\title{
Malaria Surveillance — United States, 2016
}

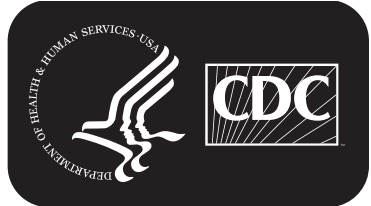




\section{CONTENTS}

Introduction

The MMWR series of publications is published by the Center for Surveillance, Epidemiology, and Laboratory Services, Centers for Disease Control and Prevention (CDC), U.S. Department of Health and Human Services, Atlanta, GA 30329-4027.

Suggested citation: [Author names; first three, then et al., if more than six.] [Title]. MMWR Surveill Summ 2019;68(No. SS-\#):[inclusive page numbers].

\section{Centers for Disease Control and Prevention \\ Robert R. Redfield, MD, Director}

Anne Schuchat, MD, Principal Deputy Director

Chesley L. Richards, MD, MPH, Deputy Director for Public Health Science and Surveillance

Rebecca Bunnell, PhD, MEd, Director, Office of Science

Barbara Ellis, PhD, MS, Acting Director, Office of Science Quality, Office of Science

Michael F. Iademarco, MD, MPH, Director, Center for Surveillance, Epidemiology, and Laboratory Services

\section{MMWR Editorial and Production Staff (Serials)}

Charlotte K. Kent, PhD, MPH, Editor in Chief Christine G. Casey, MD, Editor

Mary Dott, MD, MPH, Online Editor

Terisa F. Rutledge, Managing Editor

David C. Johnson, Lead Technical Writer-Editor

Catherine B. Lansdowne, MS, Project Editor

Matthew L. Boulton, MD, MPH

Virginia A. Caine, MD

Katherine Lyon Daniel, $\mathrm{PhD}$

Jonathan E. Fielding, MD, MPH, MBA

David W. Fleming, MD

William E. Halperin, MD, DrPH, MPH
Martha F. Boyd, Lead Visual Information Specialist Maureen A. Leahy, Julia C. Martinroe, Stephen R. Spriggs, Tong Yang, Visual Information Specialists Quang M. Doan, MBA, Phyllis H. King, Terraye M. Starr, Moua Yang,
MMWR Editorial Board

Timothy F. Jones, MD, Chairman

Robin Ikeda, MD, MPH

Phyllis Meadows, $\mathrm{PhD}, \mathrm{MSN}, \mathrm{RN}$

Jewel Mullen, MD, MPH, MPA

Jeff Niederdeppe, $\mathrm{PhD}$

Patricia Quinlisk, MD, MPH
Stephen C. Redd, MD

Patrick L. Remington, MD, MPH

Carlos Roig, MS, MA

William Schaffner, MD

Morgan Bobb Swanson, BS 


\title{
Malaria Surveillance - United States, 2016
}

\author{
Kimberly E. Mace, $\mathrm{PhD}^{1}$; Paul M. Arguin, $\mathrm{MD}^{1}$; Naomi W. Lucchi, $\mathrm{PhD}^{1}$; Kathrine R. Tan, $\mathrm{MD}^{1}$ \\ ${ }^{1}$ Malaria Branch, Division of Parasitic Diseases and Malaria, Center for Global Health, CDC
}

\begin{abstract}
Problem/Condition: Malaria in humans is caused by intraerythrocytic protozoa of the genus Plasmodium. These parasites are transmitted by the bite of an infective female Anopheles species mosquito. The majority of malaria infections in the United States occur among persons who have traveled to regions with ongoing malaria transmission. However, malaria is occasionally acquired by persons who have not traveled out of the country through exposure to infected blood products, congenital transmission, laboratory exposure, or local mosquitoborne transmission. Malaria surveillance in the United States is conducted to provide information on its occurrence (e.g., temporal, geographic, and demographic), guide prevention and treatment recommendations for travelers and patients, and facilitate transmission control measures if locally acquired cases are identified.
\end{abstract}

Period Covered: This report summarizes confirmed malaria cases in persons with onset of illness in 2016 and summarizes trends in previous years.

Description of System: Malaria cases diagnosed by blood film microscopy, polymerase chain reaction, or rapid diagnostic tests are reported to local and state health departments by health care providers or laboratory staff members. Case investigations are conducted by local and state health departments, and reports are transmitted to CDC through the National Malaria Surveillance System (NMSS), the National Notifiable Diseases Surveillance System (NNDSS), or direct CDC consultations. CDC reference laboratories provide diagnostic assistance and conduct antimalarial drug resistance marker testing on blood samples submitted by health care providers or local or state health departments. This report summarizes data from the integration of all NMSS and NNDSS cases, CDC reference laboratory reports, and CDC clinical consultations.

Results: CDC received reports of 2,078 confirmed malaria cases with onset of symptoms in 2016, including two congenital cases, three cryptic cases, and one case acquired through blood transfusion. The number of malaria cases diagnosed in the United States has been increasing since the mid-1970s. However, in 2015 a decrease occurred in the number of cases, specifically from the region of West Africa, likely due to altered travel related to the Ebola virus disease outbreak. The number of confirmed malaria cases in 2016 represents a 36\% increase compared with 2015, and the 2016 total is 153 more cases than in 2011, which previously had the highest number of cases (1,925 cases). In 2016, a total of 1,729 cases originated from Africa, and 1,061 (61.4\%) of these came from West Africa. P. falciparum accounted for the majority of the infections (1,419 [68.2\%]), followed by $P$. vivax (251 [12.1\%]). Fewer than $2 \%$ of patients were infected by two species (23 [1.1\%]). The infecting species was not reported or was undetermined in $10.8 \%$ of cases. CDC provided diagnostic assistance for $12.1 \%$ of confirmed cases and tested $10.8 \%$ of specimens with $P$. falciparum infections for antimalarial resistance markers. Of the U.S. resident patients who reported reason for travel, $69.4 \%$ were travelers who were visiting friends and relatives. The proportion of U.S. residents with malaria who reported taking any chemoprophylaxis in $2016(26.3 \%)$ was similar to that in $2015(26.6 \%)$, and adherence was poor among those who took chemoprophylaxis. Among the 964 U.S. residents with malaria for whom information on chemoprophylaxis use and travel region were known, $94.0 \%$ of patients with malaria did not adhere to or did not take a CDC-recommended chemoprophylaxis regimen. Among 795 women with malaria, 50 were pregnant, and one had adhered to mefloquine chemoprophylaxis. Forty-one (2.0\%) malaria cases occurred among U.S. military personnel in 2016, a comparable proportion to that in 2015 (23 cases [1.5\%]). Among all reported cases in 2016, a total of 306 (14.7\%) were classified as severe illnesses, and seven persons died. In 2016, CDC analyzed 144 P. falciparum-positive and nine $P$. falciparum mixed species samples for surveillance of antimalarial resistance markers (although certain loci were untestable in some samples); genetic polymorphisms associated with resistance to pyrimethamine were identified in $142(97.9 \%)$, to sulfadoxine in 98 (70.5\%), to chloroquine in 67 (44.7\%), to mefloquine in six (4.3\%), and to atovaquone in one $(<1.0 \%)$. The completeness of key variables (e.g., species, country of acquisition, and resident status) was $79.4 \%$ in 2016 and $75.7 \%$ in 2015.

Corresponding author: Kimberly E. Mace, Malaria Branch, Division of Parasitic Diseases and Malaria, Center for Global Health. Telephone: 404-718-4702; E-mail: igd3@cdc.gov. 
Interpretation: The number of reported malaria cases in 2016 continued a decades-long increasing trend and is the highest since 1972. The importation of malaria reflects the overall increase in global travel trends to and from areas where malaria is endemic; a transient decrease in the acquisition of cases, predominantly from West Africa, occurred in 2015. In 2016, more cases (absolute number) originated from regions of the world with widespread malaria transmission. Since the early 2000s, worldwide interventions to reduce malaria have been successful; however, progress has plateaued in recent years, the disease remains endemic in many regions, and the use of appropriate prevention measures by travelers remains inadequate.

Public Health Actions: The best way to prevent malaria is to take chemoprophylaxis medication during travel to a country where malaria is endemic. Malaria infections can be fatal if not diagnosed and treated promptly with antimalarial medications appropriate for the patient's age and medical history, the likely country of malaria acquisition, and previous use of antimalarial chemoprophylaxis. In 2018, two tafenoquine-based antimalarials were approved by the Food and Drug Administration (FDA) for use in the United States. Arakoda was approved for use by adults for chemoprophylaxis and is available as a weekly dosage that is convenient during travel, which might improve adherence and also can prevent relapses from $P$. vivax and $P$. ovale infections. Krintafel was approved for radical cure of $P$. vivax infections in those $>16$ years old. In April 2019, intravenous artesunate became the first-line medication for treatment of severe malaria in the United States. Because intravenous artesunate is not FDA approved, it is available from CDC under an investigational new drug protocol. Detailed recommendations for preventing malaria are available to the general public at the CDC website (https://www.cdc.gov/malaria/travelers/drugs.html). Health care providers should consult the CDC Guidelines for Treatment of Malaria in the United States and contact the CDC's Malaria Hotline for case management advice when needed. Malaria treatment recommendations are available online (https://www.cdc.gov/malaria/diagnosis_treatment) and from the Malaria Hotline (770-488-7788 or toll-free at 855-856-4713). Persons submitting malaria case reports (care providers, laboratories, and state and local public health officials) should provide complete information because incomplete reporting compromises case investigations and efforts to prevent infections and examine trends in malaria cases. Adherence to recommended malaria prevention strategies is low among U.S. travelers; reasons for nonadherence include prematurely stopping after leaving the area where malaria was endemic, forgetting to take the medication, and experiencing a side effect. Molecular surveillance of antimalarial drug resistance markers (https://www.cdc.gov/malaria/features/ars.html) enables CDC to track, guide treatment, and manage drug resistance in malaria parasites both domestically and internationally. More samples are needed to improve the completeness of antimalarial drug resistance analysis; therefore, CDC requests that blood specimens be submitted for all cases of malaria diagnosed in the United States.

\section{Introduction}

Malaria parasites of the Plasmodium genus are transmitted through the bite of infective mosquitoes. Female Anopheles species mosquitoes transmit four Plasmodium species that commonly cause illness in humans: $P$. falciparum, $P$. vivax, $P$. ovale, and $P$. malariae. Mixed infections with multiple species are possible and occur in areas where more than one species is in circulation (1). Rarely, humans can be infected with P. knowlesi, a predominantly simian malaria found in Southeast Asia.

In 2016, malaria was endemic in 91 countries and territories in the tropics and subtropics, with approximately half the world population at risk for infection. Although the true number of malaria illnesses is difficult to know with certainty, the World Health Organization (WHO) estimated that 216 million cases of malaria occurred worldwide in 2016, resulting in an estimated 445,000 deaths (2). Since 2000, the global community has funded and implemented malaria control efforts and achieved significant decreases in numbers of cases, including the prevention of millions of malaria deaths. However, since 2014, a modest but steady increase has occurred in the number of malaria cases worldwide (3).
P. falciparum and P. vivax contribute the most morbidity worldwide. P. falciparum is the most pathogenic malaria species, has the highest prevalence in sub-Saharan Africa, and is most commonly associated with severe illness and death, typically among children aged $<5$ years. The African region accounts for an estimated $90 \%$ of malaria cases worldwide; $99 \%$ of cases in Africa are due to $P$. falciparum, and $91 \%$ of all malaria deaths occur in Africa (2). P. vivax has a broader geographic range than $P$. falciparum, and outside of the African region, $P$. vivax is estimated to account for $64 \%$ of malaria infections in the Americas, 30\% of infections in Southeast Asia, and $40 \%$ of infections in the Eastern-Mediterranean region (2). In some temperate regions, $P$. vivax infections can have incubation periods of $\geq 6$ months between inoculation and onset of the first symptoms (4-6). Malaria relapses are common with $P$. vivax and $P$. ovale parasites, which have dormant liver stages (hypnozoites) that can reactivate months or years after the acute infection. P. malariae parasites are found throughout the tropics and subtropics, they account for a minority of the Plasmodium parasites in circulation, and they are often 
detected in mixed-species infections. P. malariae parasites mature slowly in human and mosquito hosts, and although they do not typically cause severe symptoms in humans, they can result in persistent low-density infections that can last for years or even a lifetime, providing opportunities for ongoing transmission $(7,8)$.

Although the Anopheles mosquito vector exists in many states (9), malaria was eliminated from the United States* in the 1950s (10). Since 1957, malaria surveillance has been supported to detect cases and prevent reintroduction, monitor antimalarial resistance, assess trends in case acquisition, and guide malaria prevention and treatment recommendations for U.S. residents. Most malaria cases diagnosed in the United States are imported from countries with ongoing mosquitoborne transmission. Occasionally, congenital transmission occurs or induced cases result from exposure to blood products. Before 2016, the last reported case of transfusion-acquired malaria had occurred in 2011 (11). State and local health departments and CDC investigate cryptic cases for which exposure cannot be explained. During 1957-2003, a total of 63 malaria outbreaks occurred in the United States. The last well-documented local mosquitoborne transmission occurred in 2003, when eight cases were diagnosed in Palm Beach, Florida (12-14).

Clinical illness results from the presence of an asexual, intraerythrocytic stage of the parasite in red blood cells; symptom severity ranges from absent or mild symptoms to severe illness and death. Factors that contribute to variability in illness severity are complex and include the parasite species, the patient's age and immune response to the infection, the presence of acquired or induced immunity, the patient's general health and nutritional constitution, chemoprophylaxis effects, and time to initiate appropriate treatment (7). Although malaria symptoms vary, the majority of patients have fever (15). Symptoms associated with uncomplicated malaria include chills, sweating, headache, fatigue, myalgia, cough, nausea, and mild anemia. If not treated promptly, malaria can affect multiple organ systems and result in altered consciousness (cerebral malaria), seizures, severe anemia, acute kidney injury and liver failure, respiratory distress, coma, permanent disability, and death. Travel history should be routinely requested for patients with fever. Malaria should be considered in the differential diagnosis for all persons who have fever and who recently traveled to areas where malaria is endemic, as well as for persons who have fever of unknown origin, regardless of travel history.

To prevent malaria, CDC recommends that U.S. residents use chemoprophylaxis: antimalarial medication taken before, during, and after travel to a country with malaria transmission.

\footnotetext{
*The term United States includes all states and territories.
}

Persons who intend to travel should see their doctor for a prescription for an antimalarial that is appropriate for the region of travel, the age of the patient, pregnancy status, and individual preferences (e.g., cost or regimen type [daily or weekly]). CDC provides chemoprophylaxis guidelines to health care providers and the public, and links to these resources are provided in this report.

This report summarizes malaria cases reported to CDC with onset of symptoms in 2016, describes trends during previous years, and highlights information on risk factors and prevention. The intended audience includes public health authorities, health care providers, persons traveling to areas with malaria transmission, and other interested readers. Information on chemoprophylaxis, diagnosis, and treatment is provided for health care professionals and the public, and links to additional malaria information and resources are provided.

\section{Methods}

\section{Data Sources and Analysis}

Malaria case reports were submitted to CDC through the National Malaria Surveillance System (NMSS) and the National Notifiable Diseases Surveillance System (NNDSS) (16). Both systems rely on passive reporting, and the number of cases might differ (e.g., because of differences in date classifications). NNDSS report dates are assigned according to the date reported to the health department, and NMSS assigns dates according to illness onset. In addition, NNDSS provides only basic case demographic information, whereas NMSS collects detailed epidemiologic data, including laboratory confirmation, travel history, and clinical history, which facilitate investigation and classification of each case. Typically, NMSS cases are reported by health care providers or laboratories to local or state health departments and then to CDC. Some cases also are reported through direct consultation with CDC staff via the Malaria Hotline or sent to CDC directly from clinics, health care providers, and laboratories. Diagnostic confirmation of cases often is facilitated by the CDC reference laboratory. The Armed Forces Health Surveillance Branch provides reports of military malaria to NMSS. This report summarizes data from the integration of all NMSS and NNDSS cases and CDC reference laboratory reports after deduplication and reconciliation.

Malaria cases are classified as confirmed or suspected using the 2014 case definition from the Council of State and Territorial Epidemiologists and CDC (17). Malaria cases are further categorized by infecting species: P. falciparum, P. vivax, $P$. malariae, and $P$. ovale. When more than a single species is detected, the case is categorized as a mixed infection. All 
categories are mutually exclusive. Diagnosis of malaria is made by blood film microscopy or polymerase chain reaction (PCR). A rapid diagnostic test (RDT) can be used to detect malaria antigens (18); however, the diagnosis must be confirmed either by microscopy or PCR to be counted as a case. Only data from confirmed cases are included in this report.

CDC staff members review all reports when received and request any additional information needed from the provider or the jurisdiction. Rare cases classified as acquired in the United States are investigated further, as are those classified as induced, congenital, introduced, or cryptic according to the definitions that follow. Instructions for completing the malaria case report form, including the applicable definitions, are available from the CDC malaria website (19). Information from the structured malaria case report form is entered into a database by a subject-matter expert dedicated to malaria surveillance (20). Data elements analyzed include age, sex, pregnancy status, residence, illness onset date, laboratory results (test type, species, and parasitemia percentage), travel history (countries, regions, and dates), chemoprophylaxis (medication used and adherence), history of malaria (date and species), blood transfusion or organ transplant history, clinical complications, treatment medications, illness outcome (survived versus died), and case classification. Records with missing data were excluded from analysis.

The chi-square test was used to calculate $p$ values and assess differences between variables reported in 2016 compared with previous years. A p value of $<0.05$ was considered statistically significant. Linear regression using the least-squares methods was used to assess the linear trend in the number of cases during 1973-2016. The Pearson product-moment correlation coefficient $\left(\mathrm{R}^{2}\right)$ was used to describe the proportion of variation explained by the model.

\section{Definitions}

The following definitions are used in malaria surveillance for the United States $(17,20)$ :

- Adherence to chemoprophylaxis: Self-reported response (yes or no) to the question, "Was chemoprophylaxis taken as prescribed?"

- Confirmed case: Symptomatic or asymptomatic infection that occurs in a person in the United States or one of its territories who has laboratory-confirmed (by microscopy or PCR) malaria parasitemia, regardless of whether the person had previous episodes of malaria while in other countries. A subsequent episode of malaria is counted as an additional case, regardless of the detected Plasmodium species, unless the case is indicated as a treatment failure within 4 weeks of initial presentation.
- Laboratory criteria for diagnosis: Demonstration of malaria parasites by microscopy or PCR (including as confirmation of an initial positive RDT).

- Non-U.S. residents: Persons who are residents of a country other than the United States.

- Suspect case: Symptomatic or asymptomatic infection that occurs in a person in the United States or one of its territories who has Plasmodium species infection detected by RDT without confirmation by microscopy or PCR, regardless of whether the person experienced previous episodes of malaria while in other countries.

- Travelers visiting friends and relatives (VFR): Immigrants, ethnically and racially distinct from the major population of the country of residence (a country where malaria is not endemic), who return to their homeland (a country where malaria is endemic) to visit friends and relatives; family members of immigrants (e.g., spouse or children) are included in this group, even if they were born in the country of residence (21). Non-U.S. residents visiting friends and relatives in the United States also are classified as VFR travelers; however, characteristics of these persons are assessed separately from U.S. resident VFR travelers.

- Treatment according to CDC recommendations (i.e., appropriate treatment): Treated with a CDCrecommended regimen appropriate for species, region, and severity of disease (22). Patients who received more antimalarial medication than recommended were classified as appropriately treated because the precise sequence and circumstances of excess treatment are not included in the malaria case report, and characterizing the purpose or appropriateness of the additional antimalarial treatment is often not possible.

- U.S. civilians: Any U.S. residents, excluding U.S. military personnel.

- U.S. residents: Persons who live in the United States, including both civilian and U.S. military personnel, regardless of legal citizenship.

This report also uses terms derived from WHO recommendations (23) and the CDC Yellow Book (24). Definitions of the following terms are included for reference:

- Congenital malaria: Malaria infection transmitted directly from mother to child during pregnancy or childbirth.

- Cryptic malaria: A case of malaria for which epidemiologic investigations cannot identify a plausible mode of acquisition.

- Imported malaria: Malaria acquired outside a specific area. In this report, imported cases are those acquired outside the United States and its territories. 
- Indigenous malaria: Local mosquitoborne transmission of malaria with no evidence of importation and no direct link to transmission from an imported case.

- Induced malaria: Malaria transmission through a blood transfusion, organ transplantation, or another parenteral route, not mosquitoborne or congenital transmission.

- Introduced malaria: Local mosquitoborne transmission of malaria with strong epidemiological evidence linking the case to an imported case.

- Radical treatment (or radical cure): Treatment to kill dormant liver-stage parasites of $P$. vivax and $P$. ovale to prevent relapses of malaria.

- Relapsing malaria: Recurrence of disease after it has been apparently cured. In malaria, true relapses are caused by activation of dormant liver-stage parasites (hypnozoites) of P. vivax and P. ovale only. Hypnozoite activation is typically delayed after the primary exposure (25); therefore, likely relapses of $P$. vivax and $P$. ovale are defined as occurring $>45$ days after travel to an area where malaria is endemic.

- Severe malaria: A case of malaria with one or more of the following manifestations: neurologic symptoms, acute kidney injury, severe anemia (hemoglobin $[\mathrm{Hb}]<7 \mathrm{~g} / \mathrm{dL}$ ), acute respiratory distress syndrome (ARDS), jaundice, or $\geq 5 \%$ parasitemia $(26,27)$. Cases also were counted as severe if the person received treatment for severe malaria (i.e., artesunate, quinidine, or an exchange blood transfusion) despite having no specific severe manifestations reported. All fatal malaria cases were classified as severe.

\section{Diagnosis of Malaria}

Three laboratory tests are available to diagnose malaria, including the peripheral blood smear, PCR, and RDT; a blood smear is recommended as the preferred first-line test. If malaria is suspected, a Giemsa-stained film of the patient's peripheral blood should be examined by microscopy for parasites as soon as possible. Microscopy allows an estimation of the level of parasitemia, which is necessary to prescribe appropriate treatment. Diagnostic accuracy depends on blood film quality and examination by experienced laboratory personnel $(28,29)$. Three sets of thick and thin blood films spaced 12-24 hours apart are needed to rule out malaria. PCR, although not readily available to be useful in the initial diagnosis and treatment of acute malaria, is used to confirm the species, which is important for $P$. vivax and $P$. ovale infections that require additional treatment to prevent relapse $(17,24)$.

The BinaxNOW malaria RDT (Inverness Medical Professional Diagnostics) detects circulating malaria-specific antigens and is approved for use by hospital and commercial laboratories; the test is not approved as a point-of-care test by clinicians or the general public $(18,30)$. RDTs are less sensitive than microscopy and not able to determine all Plasmodium species or quantify malaria parasites; therefore, the results require confirmation and species identification by microscopy (18). If microscopy is not performed, then PCR can confirm an RDT result and determine the species.

\section{Drug Resistance Marker Surveillance}

In 2012, CDC began molecular surveillance, with the goal of detecting and characterizing malaria parasites that carry genetic markers (typically single nucleotide polymorphisms [SNPs] in one or more loci) associated with drug resistance. The surveillance data are used to identify where drug-resistant foci might be present or emerging in specific parts of the world where malaria is endemic. For each sample submitted, species confirmation is conducted using real-time PCR assays capable of detecting the four primary human-infecting Plasmodium species. Molecular genotyping for known markers of drug resistance (typically SNPs and gene copy number variation) are investigated for all $P$. falciparum samples. Additional species will be similarly evaluated as new laboratory methods are developed. Each sample submitted is tested for molecular markers associated with resistance to sulfadoxine, pyrimethamine, chloroquine, mefloquine, atovaquone, and artemisinin.

Samples for molecular resistance monitoring are processed for PCR amplification of parasite DNA using appropriate primers for the genes of interest and sequenced by the Sanger method using the ABI 3130 capillary sequencer (Thermo Fisher Scientific) according to described methods (31). Fragments of genes encoding molecular targets of chloroquine (chloroquine resistance transporter gene, $p f(r t)$, pyrimethamine and proguanil (dihydrofolate reductase gene, $p f d h f r$ ), sulfadoxine (dihydropteroate synthase gene, $p f d h p s$ ), atovaquone (cytochrome b gene, $p f c y t b$ ), mefloquine (multidrug resistance 1 protein gene, $p f m d r-1$ and $p f m d r-1$ copy number), and artemisinin (kelch K13-propeller domain) are analyzed for SNPs by comparing each sequence to the reference genome.

Chloroquine resistance markers: The $p f c r t$ gene sequence is analyzed to identify polymorphisms C72S, M74I, N75E, and K76T.

Pyrimethamine and proguanil resistance markers: The $p f d h f r$ gene sequence is analyzed to identify polymorphisms A16V, C50R, N51I, C59R, S108T/N, and I164L. 
Sulfadoxine resistance markers: The $p f d h p s$ gene sequence is analyzed to identify polymorphisms S436A, A437G, and K540E.

Atovaquone resistance markers: The $p f c y t b$ gene sequence is analyzed to identify polymorphisms I258M and Y268S (32).

Mefloquine resistance markers: A real-time PCR assay is used to determine the $p f m d r-1$ copy number variation in the $P$. falciparum samples using the comparative cycle threshold $\left(\Delta \Delta \mathrm{C}_{\mathrm{T}}\right)$ method as previously described (33). DNA from the 3D7 laboratory control, which has a single copy of $p f m d r-1$, is used as the calibrator. In addition, DNA from Indochina W2mef and Dd2 are used as multiple copy number controls.

Artemisinin resistance markers: The K13-propeller domain for artemisinin resistance is amplified using a nested PCR method previously described $(34,35)$. The sequence data are analyzed using Geneious Pro R8 (Biomatters).

\section{Results}

\section{General Surveillance}

CDC received 2,078 reports of confirmed malaria cases and 10 reports of suspected cases (tested by RDT only) among persons in the United States and its territories with onset of symptoms during 2016 (Table 1), which is a $36.4 \%$ increase in confirmed malaria cases compared with $2015(\mathrm{n}=1,524)^{\dagger}$ and the highest number of cases since 1972. The highest numbers of malaria cases reported in the United States since malaria elimination in the 1950s were in 1970 and 1971, when 4,247 and 3,180 malaria cases were detected in the United States, predominantly among military personnel deployed to Southeast Asia. Then, starting from only 614 cases in 1972, the number of malaria cases has increased to an average of 1,718 annual cases during 2012-2014 (Table 1). Cases imported from Africa contributed almost all $(91 \%)$ of the increase from 2015 to 2016, with West Africa ${ }^{\$}$ accounting for 49\% of this increase. Since 1972, the overall trend has been an increasing number of malaria cases, with an average gain of

\footnotetext{
$\dagger$ The number of cases reported for 2015 is different from that published in "Malaria Surveillance — United States, 2015." This is because of delayed reporting of cases with symptom onset in late 2015 but counted as 2016 cases according to NNDSS definitions. This analysis reclassified all cases according to date of symptom onset.

${ }^{\S}$ Countries that are considered West Africa include Benin, Burkina Faso, Cape Verde, Côte d'Ivoire, Gambia, Ghana, Guinea, Guinea-Bissau, Liberia, Mali, Mauritania, Niger, Nigeria , Senegal, Sierra Leone, Togo, and West Africa, unspecified.
}

28.7 cases per year $\left(\mathrm{R}^{2}=0.727\right)$ (Figure 1$)$. This increase in malaria cases coincides with the increasing trend in the annual number of international airline flights taken by U.S. citizens (36). According to data from the National Travel and Tourism Office, during 1996-2016, an increase in travel over time occurred, with an average annual addition of approximately 555,000 travel events to all destinations $\left(\mathrm{R}^{2}=0.776\right)$, including an average annual gain of approximately 12,000 travel events to Africa $\left(\mathrm{R}^{2}=0.716\right)(37)$. In $2016,1,216(58.5 \%)$ cases were among U.S. civilians, $41(2.0 \%)$ among U.S. military members, $581(28.0 \%)$ were among non-U.S. residents, and $240(11.6 \%)$ were among patients with unknown or unreported resident status (Table 1 ).

Of the 2,078 confirmed cases, 2,056 cases were imported from countries in which malaria is endemic; two cases were classified as congenital, three remained classified as cryptic after an investigation, and one was transmitted through a blood transfusion. Sixteen case reports had an incomplete travel history that prevented classification. Of the 16 cases with unknown classification, six had laboratory reports only, and 10 were reported by local or state health departments as lost to follow-up with insufficient information to classify importation status. In 2016, 20.6\% case reports were missing one or more of the essential variables (e.g., infecting species, residence status, and country of acquisition), which is a significant improvement from $2015(24.3 \%)$. Of the confirmed cases, $10.8 \%$ were missing the infecting species, $11.5 \%$ were missing residence status, and $3.0 \%$ were missing the country of acquisition; in $2015,12.9 \%$ of case reports did not include the species, $13.1 \%$ were missing residence status, and $5.8 \%$ were missing the country of acquisition. No case in 2016 was reported as having been acquired by mosquitoborne transmission in the United States.

\section{Plasmodium Species}

In 2016, a total of $1,853(89.2 \%)$ cases reported to CDC included information on the infecting Plasmodium species (Table 2), which is similar to the proportion from 2015 (87.1\%). Specimens from $252(12.1 \%)$ cases were sent to the CDC reference laboratory for diagnostic assistance, and CDC identified the species for 243 (96.4\%) specimens. Among these 243 specimens, CDC determined or corrected the species for 186 (76.5\%). An additional 592 specimens submitted were negative for Plasmodium.

Among the 1,853 cases with a species determination, 1,419 (76.6\%) were $P$. falciparum, and 251 (13.5\%) were $P$. vivax infections (Table 2). A total of 99 (5.3\%) P. ovale, 61 (3.3\%) P. malariae, and 23 (1.2\%) mixed species infections were identified. Of the 23 mixed infections, seven were $P$. falciparum 
TABLE 1. Number of malaria cases* among U.S. military personnel, U.S. civilians, and non-U.S residents — United States, 1970-2016

\begin{tabular}{|c|c|c|c|c|c|}
\hline Year & U.S. military personnel & U.S. civilians & Non-U.S. residents & Status not recorded & Total \\
\hline 1970 & 4,096 & 90 & 44 & 17 & 4,247 \\
\hline 1971 & 2,975 & 79 & 69 & 57 & 3,180 \\
\hline 1972 & 454 & 106 & 54 & 0 & 614 \\
\hline 1973 & 41 & 103 & 78 & 0 & 222 \\
\hline 1974 & 21 & 158 & 144 & 0 & 323 \\
\hline 1975 & 17 & 199 & 232 & 0 & 448 \\
\hline 1976 & 5 & 178 & 227 & 5 & 415 \\
\hline 1977 & 11 & 233 & 237 & 0 & 481 \\
\hline 1978 & 31 & 270 & 315 & 0 & 616 \\
\hline 1979 & 11 & 229 & 634 & 3 & 877 \\
\hline 1980 & 26 & 303 & 1,534 & 1 & 1,864 \\
\hline 1981 & 21 & 273 & 809 & 0 & 1,103 \\
\hline 1982 & 8 & 348 & 574 & 0 & 930 \\
\hline 1983 & 10 & 325 & 468 & 0 & 803 \\
\hline 1984 & 24 & 360 & 632 & 0 & 1,016 \\
\hline 1985 & 31 & 446 & 568 & 0 & 1,045 \\
\hline 1986 & 35 & 410 & 646 & 0 & 1,091 \\
\hline 1987 & 23 & 421 & 488 & 0 & 932 \\
\hline 1988 & 33 & 550 & 440 & 0 & 1,023 \\
\hline 1989 & 35 & 591 & 476 & 0 & 1,102 \\
\hline 1990 & 36 & 558 & 504 & 0 & 1,098 \\
\hline 1991 & 22 & 585 & 439 & 0 & 1,046 \\
\hline 1992 & 29 & 394 & 481 & 6 & 910 \\
\hline 1993 & 278 & 519 & 453 & 25 & 1,275 \\
\hline 1994 & 38 & 524 & 370 & 82 & 1,014 \\
\hline 1995 & 12 & 599 & 461 & 95 & 1,167 \\
\hline 1996 & 32 & 618 & 636 & 106 & 1,392 \\
\hline 1997 & 28 & 698 & 592 & 226 & 1,544 \\
\hline 1998 & 22 & 636 & 361 & 208 & 1,227 \\
\hline 1999 & 55 & 833 & 381 & 271 & 1,540 \\
\hline 2000 & 46 & 827 & 354 & 175 & 1,402 \\
\hline 2001 & 18 & 891 & 316 & 158 & 1,383 \\
\hline 2002 & 33 & 849 & 272 & 183 & 1,337 \\
\hline 2003 & 36 & 767 & 306 & 169 & 1,278 \\
\hline 2004 & 32 & 775 & 282 & 235 & 1,324 \\
\hline 2005 & 36 & 870 & 297 & 325 & 1,528 \\
\hline 2006 & 50 & 736 & 217 & 561 & 1,564 \\
\hline 2007 & 33 & 701 & 263 & 508 & 1,505 \\
\hline 2008 & 19 & 510 & 176 & 593 & 1,298 \\
\hline 2009 & 18 & 661 & 201 & 604 & 1,484 \\
\hline 2010 & 46 & 1,085 & 368 & 192 & 1,691 \\
\hline 2011 & 91 & 1,098 & 386 & 350 & 1,925 \\
\hline 2012 & 43 & 1,121 & 328 & 195 & 1,687 \\
\hline 2013 & 14 & 1,136 & 349 & 242 & 1,741 \\
\hline 2014 & 31 & 1,114 & 384 & 196 & 1,725 \\
\hline $2015^{\dagger}$ & 23 & 933 & 368 & 200 & 1,524 \\
\hline 2016 & 41 & 1,216 & 581 & 240 & 2,078 \\
\hline
\end{tabular}

* A case was defined as symptomatic or asymptomatic illness that occurs in the United States or one of its territories in a person who has laboratory-confirmed (by microscopy or polymerase chain reaction) malaria parasitemia, regardless of whether the person previously had malaria while in other countries. A subsequent case of malaria occurring in a person is counted as an additional case if the demonstrated Plasmodium species differs from the initially identified species or if it is indicated as a relapsing infection demonstrating the same Plasmodium species as identified previously. If a subsequent episode of malaria occurs as a result of a drug failure caused by a resistant parasite, then it is not counted as an additional case.

† 2015 data were updated to include cases reported after publication of the initial data in 2015 (Mace KE, Arguin PM, Tan KR. Malaria surveillance-United States, 2015. MMWR Surveill Summ 2018;67[No. SS-7]).

with $P$. ovale, seven were $P$. falciparum with $P$. malariae, five were $P$. falciparum with $P$. vivax, two were $P$. vivax with $P$. ovale, one was $P$. ovale with $P$. malariae, and one was $P$. falciparum, $P$. ovale, and $P$. malariae. Twenty of 23 mixed infections were PCR confirmed, nine of these by the CDC reference laboratory, including the specimen with three Plasmodium species. The species distribution in 2016 for $P$. falciparum, P. vivax, P. ovale,
P. malariae ${ }_{s}$ mixed Plasmodium species, and unknown species infections was similar to that in 2015.

In 2016, a total of 717 (34.5\%) malaria infections reported in the United States were confirmed by PCR, an increase from 2015, when 408 of 1,524 (26.8\%) infections were PCR confirmed. Approximately one third of the 1,419 P. falciparum infections and $251 P$. vivax infections were PCR confirmed 


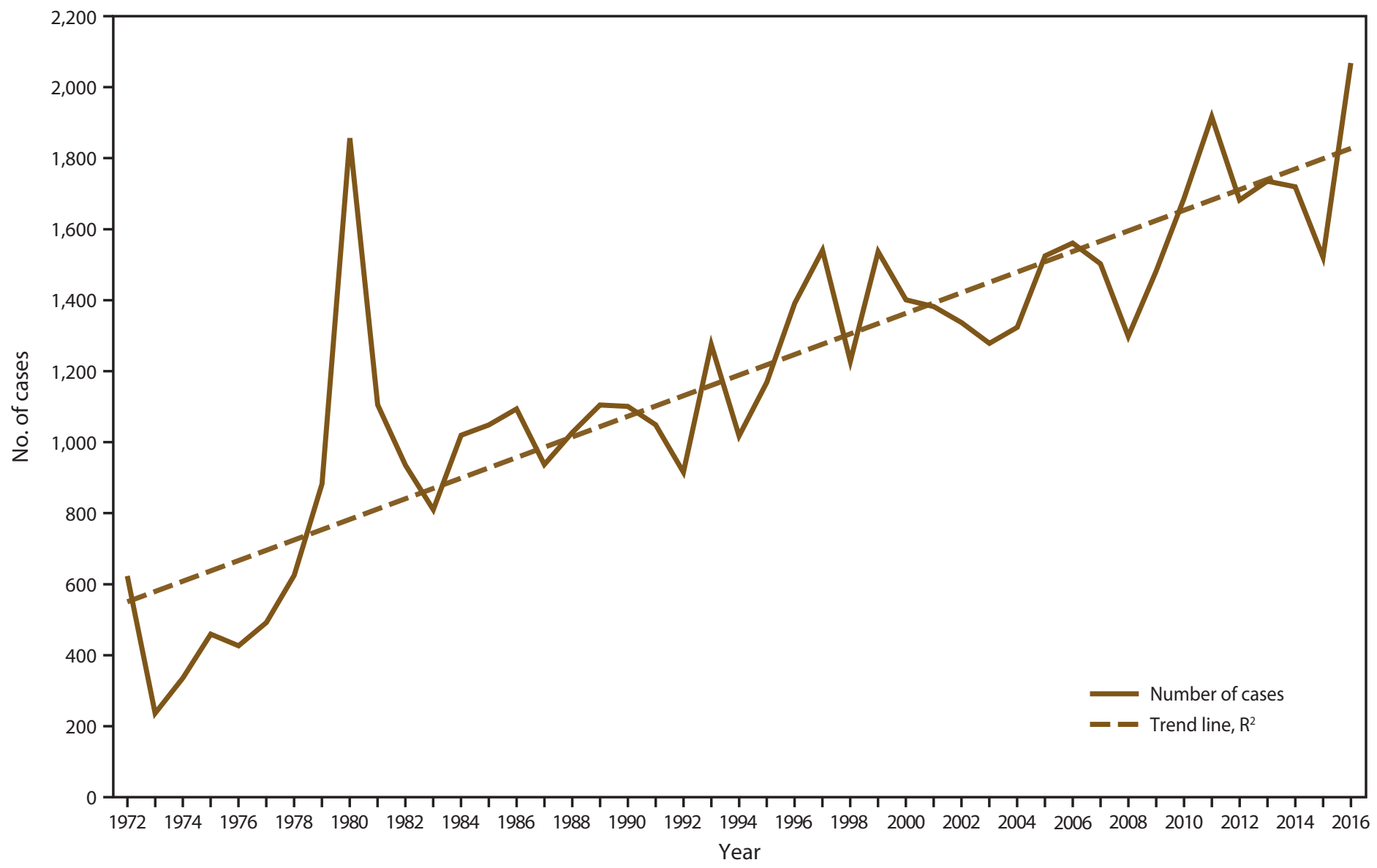

Abbreviation: $\mathrm{R}^{2}$ = square of the Pearson product moment correlation coefficient. ${ }^{*} R^{2}=0.694$ is the average rate in increase of cases over time.

(501 [35.3\%] and 91 [36.3\%], respectively). Of 61 P. malariae cases, 25 (41.0\%) were PCR confirmed; 63 (63.6\%) of 99 P. ovale infections were PCR confirmed, and 20 (87.0\%) of 23 mixed species infections were PCR confirmed.

In early 2017, U. S. refugee health coordinators informed CDC that they diagnosed numerous $P$. malariae cases among refugees of Congolese nationality who had received predeparture treatment with artemether-lumefantrine (38). From 2015 to 2016, no significant increase occurred in P. malariae cases among all refugees and immigrants $(\mathrm{n}=8$ [6.6\%] in 2015 and $\mathrm{n}=21$ [7.9\%] in 2016). However, an increase in $P$. malariae cases occurred among refugees and immigrants from the Democratic Republic of Congo, Uganda, Tanzania, and the Central African Republic (3 [6.3\%] in 2015 and 19 [31.2\%] in 2016); eight of the 19 P. malariae cases in 2016 were PCR confirmed.

\section{Region of Acquisition and Diagnosis}

Information on the region of acquisition was available for $2,016(98.1 \%)$ of 2,056 imported cases; of these 1,729 (85.8\%) were from Africa, 174 (8.6\%) from Asia, 70 (3.5\%) from Central America and the Caribbean, 37 (1.8\%) from South America, and six $(<1.0 \%)$ from Oceania (Table 3$)$.

Of the 1,387 P. falciparum cases reported in 2016 with a known region of acquisition, 1,335 (96.3\%) were acquired from Africa, $35(2.5 \%)$ from Central America and the Caribbean, $15(1.1 \%)$ from Asia, and one case each from South America and Oceania $(<1 \%)$. P. falciparum infections accounted for $85.0 \%$ of cases from Africa, $10.2 \%$ from Asia, $57.4 \%$ from Central America and the Caribbean, 3.7\% from South America, and 20.0\% from Oceania (Table 3).

In 2016, a total of 245 imported $P$. vivax infections were reported with information about region of acquisition. Of these, 127 (51.8\%) were acquired from Asia, followed by 65 (26.5\%) from Africa, 24 (9.8\%) from Central America and the Caribbean, 25 (10.2\%) from South America, and four (1.6\%) from Oceania. In 2016, a total of 96 P. ovale and 
TABLE 2. Number of malaria cases, by Plasmodium species and year — United States, 2012-2016

\begin{tabular}{|c|c|c|c|c|c|c|c|c|c|c|}
\hline \multirow[b]{2}{*}{ Plasmodium species } & \multicolumn{2}{|c|}{2012} & \multicolumn{2}{|l|}{2013} & \multicolumn{2}{|l|}{2014} & \multicolumn{2}{|c|}{$2015^{*}$} & \multicolumn{2}{|c|}{2016} \\
\hline & No. $(\%)^{\dagger}$ & $(\%)^{\S}$ & No. (\%) & $(\%)$ & No. $(\%)$ & (\%) & No. (\%) & (\%) & No. $(\%)$ & $(\%)$ \\
\hline P. falciparum & $985(58.4)$ & $(70.4)$ & $1,059(60.8)$ & $(72.8)$ & $1,141(66.1)$ & $(74.9)$ & $1,025(67.3)$ & $(77.2)$ & $1,419(68.3)$ & (76.6) \\
\hline P. vivax & $280(16.6)$ & $(20.0)$ & $245(14.1)$ & (16.8) & $230(13.3)$ & (15.1) & $180(11.8)$ & (13.6) & $251(12.1)$ & (13.5) \\
\hline P. ovale & $59(3.5)$ & $(4.2)$ & 65 (3.7) & (4.5) & $90(5.2)$ & (5.9) & $63(4.1)$ & (4.7) & $99(4.8)$ & (5.3) \\
\hline P. malariae & $54(3.2)$ & (3.9) & $45(2.6)$ & (3.1) & $47(2.8)$ & (3.1) & $48(3.2)$ & (3.6) & $61(2.9)$ & (3.3) \\
\hline Mixed & $21(1.2)$ & (1.5) & $41(2.3)$ & (2.8) & $15(0.9)$ & $(1.0)$ & $12(0.8)$ & (0.9) & $23(1.1)$ & $(1.2)$ \\
\hline Undetermined & $288(17.1)$ & NA & $286(16.4)$ & NA & $202(11.7)$ & NA & $196(12.9)$ & NA & $225(10.8)$ & NA \\
\hline Total & $1,687(100)$ & $(100)$ & $1,741(100)$ & $(100)$ & $1,725(100)$ & $(100)$ & $1,524(100)$ & (100) & $2,078(100)$ & (100) \\
\hline
\end{tabular}

Abbreviation: $\mathrm{NA}=$ not applicable.

* 2015 data were updated to include cases reported after publication of the initial data in 2015 (Mace KE, Arguin PM, Tan KR. Malaria surveillance-United States, 2015. MMWR Surveill Summ 2018;67[No. SS-7]).

† Percentage among all infections.

$\S$ Percentage among infections with known species.

59 P. malariae infections were reported with information about the region of acquisition; 93 (96.9\%) and 55 (93.2\%) were acquired in Africa, respectively.

Overall, 514 more cases were acquired from Africa in 2016 than in 2015 ( 1,729 cases in 2016 versus 1,215 cases in 2015). In 2016, the number of cases acquired from West Africa was 1,061 (61.4\% of cases from Africa), although the percentage was not significantly different from that in 2015 (784 cases, 64.5\%). In 2016 compared with 2015, more cases were imported from the following African countries: Nigeria (153 more), Sierra Leone (93 more), Uganda (67 more), Democratic Republic of the Congo (44 more), Cameroon (38 more), Liberia (30 more), Tanzania (27 more), Ethiopia (22 more), Guinea (18 more), and Burkina Faso (12 more). Angola, Gabon, Rwanda, Niger, Benin, and Chad, all had five to 10 more cases in 2016 than in 2015. Countries in Africa with fewer cases imported into the United States in 2016 than in 2015 include Ivory Coast (28 fewer), Ghana (22 fewer), Malawi (five fewer), and Kenya (five fewer).

Although the number of cases imported from Asia increased by 50 cases (a $40.3 \%$ increase) in 2016, the proportion of cases from Asia in 2016 was the same as in 2015 (174 [8.6\%] and 124 [8.6\%], respectively). The greatest number of cases imported from this region were from India, with 65 cases from India in 2015 and 89 in 2016. The number of cases imported from Afghanistan was 20 in 2015 and 39 in 2016, and the number of infections imported from Pakistan was 27 in 2015 and 20 in 2016. Two cases were acquired from South Korea in 2015 and 10 were acquired in 2016. Two cases were acquired from Indonesia in 2015 and seven in 2016. Fewer than five cases each were acquired from Cambodia, Laos, Philippines, and Thailand in 2016.

The proportion of imported cases from Central and South America and the Caribbean were not significantly different in 2016 (107 cases [5.3\%]) and 2015 (93 cases [6.5\%]). However, the number of cases imported from the Caribbean was lower in 2016 (35 cases [1.7\%]) than in 2015 (44 cases [3.1\%]).
Thirty-seven cases (1.8\%) were from South America in 2016, and 26 cases (1.8\%) were from this region in 2015. In 2016 and $2015,35(1.7 \%)$ cases and $23(1.6 \%)$ cases, respectively, were from Central America.

Thirty-two of 35 cases from the Caribbean region (Haiti and the Dominican Republic) were P. falciparum species; three cases were reported as unknown species. The number of cases from Haiti decreased from a peak of 171 cases in 2010, to 12 in 2015; in 2016, the number of malaria cases from Haiti was 22 (not a significant increase compared with 2015). Eighteen of 22 patients provided a reason for travel to Haiti, 17 traveled to visit friends and relatives, one traveled for military reasons. Five (22.7\%) of 22 patients who acquired malaria from Haiti had severe illness. During 2005-2015, the United States had an average of 4.6 cases per year imported from the Dominican Republic. In 2014, two cases were imported from the Dominican Republic, and the number increased significantly to 32 cases in 2015; in 2016, the number of cases from the Dominican Republic decreased to 13. Seven of 13 case reports indicated tourism as the reason for travel to the Dominican Republic, and two case reports indicated travel for medical procedures; four case reports did not have a reason for travel reported. Two (15.4\%) of 13 patients who acquired malaria from the Dominican Republic had severe illness.

Peru, Guyana, and Brazil were the source of the majority of the 37 cases reported from South America (12, 11, and seven cases, respectively). Twenty-five of 37 infections acquired in South America were $P$. vivax, one each were $P$. falciparum and $P$. ovale, and 10 did not have infecting species determined. Twenty-five patients who traveled to South America provided a reason for travel: 10 traveled to visit friends and relatives, seven traveled for tourism, four for education (student or teacher), two for missionary work, and one for business. One patient had severe P. vivax illness acquired from South America.

In Central America, the majority of the 35 cases were acquired in Honduras (12 cases), Guatemala (11 cases), and Central America (nine cases, unspecified country of origin); 
TABLE 3. Total number of imported malaria cases and cases confirmed by polymerase chain reaction, by country of acquisition and Plasmodium species - United States, 2016

\begin{tabular}{|c|c|c|c|c|c|c|c|c|c|c|c|c|c|c|}
\hline \multirow[b]{2}{*}{ Country of acquisition } & \multicolumn{2}{|c|}{ P. falciparum } & \multicolumn{2}{|c|}{ P. vivax } & \multicolumn{2}{|c|}{ P. ovale } & \multicolumn{2}{|c|}{ P. malariae } & \multicolumn{2}{|c|}{ Mixed } & \multicolumn{2}{|c|}{ Unknown } & \multicolumn{2}{|c|}{ Total } \\
\hline & PCR & Total & PCR & Total & PCR & Total & PCR & Total & PCR & Total & PCR & Total & PCR & Total \\
\hline Africa & 481 & 1,335 & 15 & 65 & 59 & 93 & 25 & 55 & 19 & 22 & 12 & 159 & 611 & 1,729 \\
\hline Angola & 4 & 15 & 0 & 0 & 0 & 0 & 0 & 0 & 0 & 0 & 0 & 0 & 4 & 15 \\
\hline Benin & 3 & 9 & 0 & 0 & 0 & 0 & 0 & 0 & 0 & 0 & 0 & 0 & 3 & 9 \\
\hline Burkina Faso & 14 & 29 & 0 & 0 & 0 & 1 & 0 & 1 & 2 & 2 & 0 & 3 & 16 & 36 \\
\hline Burundi & 0 & 3 & 0 & 0 & 0 & 0 & 0 & 0 & 1 & 1 & 0 & 0 & 1 & 4 \\
\hline Cameroon & 21 & 93 & 1 & 3 & 3 & 8 & 3 & 3 & 3 & 3 & 1 & 12 & 32 & 122 \\
\hline Central African Republic & 4 & 7 & 0 & 0 & 0 & 0 & 0 & 0 & 0 & 0 & 0 & 0 & 4 & 7 \\
\hline Chad & 4 & 9 & 0 & 2 & 1 & 2 & 0 & 0 & 0 & 1 & 0 & 1 & 5 & 15 \\
\hline Congo, Republic of & 16 & 62 & 0 & 1 & 1 & 2 & 3 & 6 & 1 & 1 & 1 & 6 & 22 & 78 \\
\hline Djibouti & 2 & 2 & 0 & 0 & 0 & 0 & 0 & 0 & 0 & 0 & 1 & 1 & 3 & 3 \\
\hline Equatorial Guinea & 2 & 4 & 0 & 0 & 0 & 0 & 0 & 0 & 0 & 0 & 0 & 1 & 2 & 5 \\
\hline Eritrea & 0 & 0 & 1 & 2 & 0 & 0 & 0 & 0 & 0 & 0 & 0 & 0 & 1 & 2 \\
\hline Ethiopia & 6 & 14 & 7 & 22 & 0 & 1 & 0 & 0 & 1 & 1 & 0 & 3 & 14 & 41 \\
\hline Gabon & 2 & 7 & 0 & 0 & 0 & 1 & 0 & 0 & 0 & 0 & 0 & 2 & 2 & 10 \\
\hline Gambia & 2 & 3 & 0 & 1 & 0 & 0 & 0 & 0 & 0 & 0 & 0 & 0 & 2 & 4 \\
\hline Ghana & 42 & 103 & 0 & 2 & 4 & 6 & 0 & 2 & 0 & 0 & 2 & 12 & 48 & 125 \\
\hline Guinea & 24 & 49 & 0 & 1 & 3 & 3 & 0 & 3 & 1 & 1 & 0 & 1 & 28 & 58 \\
\hline Guinea-Bissau & 0 & 4 & 0 & 0 & 0 & 0 & 0 & 0 & 0 & 0 & 0 & 0 & 0 & 4 \\
\hline Ivory Coast & 21 & 53 & 0 & 0 & 1 & 3 & 1 & 1 & 1 & 1 & 0 & 1 & 24 & 59 \\
\hline Kenya & 10 & 42 & 0 & 2 & 3 & 3 & 2 & 3 & 0 & 0 & 0 & 4 & 15 & 54 \\
\hline Liberia & 34 & 78 & 0 & 1 & 3 & 3 & 2 & 5 & 0 & 0 & 1 & 10 & 40 & 97 \\
\hline Madagascar & 1 & 1 & 0 & 0 & 0 & 0 & 0 & 0 & 0 & 0 & 0 & 0 & 1 & 1 \\
\hline Malawi & 3 & 7 & 0 & 1 & 0 & 0 & 0 & 0 & 0 & 0 & 0 & 1 & 3 & 9 \\
\hline Mali & 6 & 11 & 0 & 0 & 0 & 1 & 0 & 0 & 0 & 0 & 0 & 0 & 6 & 12 \\
\hline Mozambique & 4 & 7 & 0 & 0 & 0 & 0 & 0 & 0 & 0 & 0 & 0 & 1 & 4 & 8 \\
\hline Niger & 3 & 8 & 0 & 0 & 0 & 1 & 0 & 0 & 0 & 1 & 0 & 0 & 3 & 10 \\
\hline Nigeria & 123 & 363 & 0 & 6 & 21 & 27 & 4 & 9 & 4 & 4 & 2 & 37 & 154 & 446 \\
\hline Rwanda & 4 & 11 & 0 & 1 & 2 & 3 & 1 & 1 & 0 & 0 & 0 & 2 & 7 & 18 \\
\hline Senegal & 10 & 14 & 0 & 0 & 0 & 0 & 1 & 1 & 0 & 0 & 0 & 2 & 11 & 17 \\
\hline Sierra Leone & 41 & 115 & 0 & 3 & 2 & 3 & 1 & 1 & 0 & 0 & 1 & 12 & 45 & 134 \\
\hline Somali Republic & 0 & 0 & 0 & 1 & 0 & 0 & 0 & 0 & 0 & 0 & 0 & 0 & 0 & 1 \\
\hline South Africa & 2 & 3 & 0 & 0 & 0 & 0 & 0 & 0 & 0 & 0 & 0 & 1 & 2 & 4 \\
\hline South Sudan & 2 & 12 & 1 & 1 & 1 & 1 & 0 & 0 & 0 & 0 & 0 & 2 & 4 & 16 \\
\hline Sudan & 12 & 20 & 2 & 4 & 0 & 2 & 0 & 1 & 0 & 0 & 0 & 6 & 14 & 33 \\
\hline Tanzania & 7 & 31 & 0 & 1 & 3 & 5 & 1 & 4 & 0 & 0 & 1 & 9 & 12 & 50 \\
\hline Togo & 13 & 25 & 0 & 0 & 0 & 0 & 0 & 0 & 0 & 0 & 0 & 3 & 13 & 28 \\
\hline Uganda & 21 & 68 & 3 & 8 & 10 & 16 & 5 & 12 & 3 & 3 & 1 & 16 & 43 & 123 \\
\hline Zambia & 2 & 5 & 0 & 0 & 0 & 0 & 1 & 1 & 0 & 0 & 0 & 0 & 3 & 6 \\
\hline Central Africa, unspecified & 0 & 1 & 0 & 0 & 0 & 0 & 0 & 0 & 0 & 0 & 0 & 0 & 0 & 1 \\
\hline East Africa, unspecified & 2 & 9 & 0 & 1 & 0 & 0 & 0 & 1 & 1 & 1 & 0 & 5 & 3 & 17 \\
\hline South Africa, unspecified & 1 & 3 & 0 & 1 & 0 & 0 & 0 & 0 & 1 & 1 & 0 & 0 & 2 & 5 \\
\hline West Africa, unspecified & 7 & 17 & 0 & 0 & 0 & 0 & 0 & 0 & 0 & 1 & 0 & 4 & 7 & 22 \\
\hline Africa, unspecified & 6 & 18 & 0 & 0 & 1 & 1 & 0 & 0 & 0 & 0 & 1 & 1 & 8 & 20 \\
\hline
\end{tabular}

See table footnotes on next page.

one case each was reported from Mexico, Nicaragua, and Panama. Twenty-four of 35 cases were $P$. vivax, three were $P$. falciparum, and one each was $P$. malariae and $P$. ovale; six cases were reported as species not determined. One severe case (of unknown species) was acquired from Central America. Twenty-seven patients provided a reason for travel to Central America: 12 were refugees or immigrants, six were VFR travelers, three were missionaries, two each traveled for tourism or education (as a student or teacher), and one each traveled for business or another reason. Reported cases from Honduras increased from four in 2015 to 12 in 2016. Fifteen cases were reported from Guatemala in 2015 and 11 in 2016. In 2016, one $P$. ovale case was reported from Mexico, a country with limited malaria transmission and no recent history of $P$. ovale infection. The patient was a 15 -month-old boy who had lived in Juarez, Mexico, for 1 year and moved with his family back to the United States. The state public health laboratory and CDC obtained blood specimens from the patient and performed PCR testing, which confirmed $P$. ovale infection. The CDC informed the Mexico Ministry of Health, and they conducted a local investigation.

Six cases were reported from Oceania in 2016, all of them from Papua New Guinea; two cases were reported from Oceania in 2015 (also from Papua New Guinea). Four of six cases from this region in 2016 were $P$. vivax, one was $P$. falciparum, and one was reported with the species not determined. Four of six 
TABLE 3. (Continued) Total number of imported malaria cases and cases confirmed by polymerase chain reaction, by country of acquisition and Plasmodium species - United States, 2016

\begin{tabular}{|c|c|c|c|c|c|c|c|c|c|c|c|c|c|c|}
\hline \multirow[b]{2}{*}{ Country of acquisition } & \multicolumn{2}{|c|}{ P. falciparum } & \multicolumn{2}{|c|}{ P. vivax } & \multicolumn{2}{|c|}{ P. ovale } & \multicolumn{2}{|c|}{ P. malariae } & \multicolumn{2}{|c|}{ Mixed } & \multicolumn{2}{|c|}{ Unknown } & \multicolumn{2}{|c|}{ Total } \\
\hline & PCR & Total & PCR & Total & PCR & Total & PCR & Total & PCR & Total & PCR & Total & PCR & Total \\
\hline Asia & 3 & 15 & 56 & 127 & 1 & 1 & 0 & 3 & 1 & 1 & 0 & 27 & 61 & 174 \\
\hline Afghanistan & 0 & 0 & 13 & 28 & 0 & 0 & 0 & 2 & 1 & 1 & 0 & 8 & 14 & 39 \\
\hline Cambodia & 0 & 2 & 0 & 0 & 0 & 0 & 0 & 0 & 0 & 0 & 0 & 0 & 0 & 2 \\
\hline India & 1 & 7 & 31 & 66 & 1 & 1 & 0 & 1 & 0 & 0 & 0 & 14 & 33 & 89 \\
\hline Indonesia & 1 & 2 & 1 & 4 & 0 & 0 & 0 & 0 & 0 & 0 & 0 & 1 & 2 & 7 \\
\hline Korea, South & 0 & 0 & 3 & 9 & 0 & 0 & 0 & 0 & 0 & 0 & 0 & 1 & 3 & 10 \\
\hline Laos & 0 & 0 & 0 & 0 & 0 & 0 & 0 & 0 & 0 & 0 & 0 & 1 & 0 & 1 \\
\hline Pakistan & 0 & 0 & 8 & 19 & 0 & 0 & 0 & 0 & 0 & 0 & 0 & 1 & 8 & 20 \\
\hline Philippines & 0 & 1 & 0 & 0 & 0 & 0 & 0 & 0 & 0 & 0 & 0 & 0 & 0 & 1 \\
\hline Thailand & 0 & 0 & 0 & 1 & 0 & 0 & 0 & 0 & 0 & 0 & 0 & 0 & 0 & 1 \\
\hline Asia, unspecified & 0 & 1 & 0 & 0 & 0 & 0 & 0 & 0 & 0 & 0 & 0 & 0 & 0 & 1 \\
\hline Southeast Asia, unspecified & 1 & 2 & 0 & 0 & 0 & 0 & 0 & 0 & 0 & 0 & 0 & 1 & 1 & 3 \\
\hline Central America and the Caribbean & 8 & 35 & 8 & 24 & 1 & 1 & 0 & 1 & 0 & 0 & 1 & 9 & 18 & 70 \\
\hline Dominican Republic & 3 & 12 & 0 & 0 & 0 & 0 & 0 & 0 & 0 & 0 & 0 & 1 & 3 & 13 \\
\hline Guatemala & 0 & 0 & 3 & 8 & 0 & 0 & 0 & 1 & 0 & 0 & 0 & 2 & 3 & 11 \\
\hline Haiti & 4 & 20 & 0 & 0 & 0 & 0 & 0 & 0 & 0 & 0 & 1 & 2 & 5 & 22 \\
\hline Honduras & 1 & 2 & 4 & 9 & 0 & 0 & 0 & 0 & 0 & 0 & 0 & 1 & 5 & 12 \\
\hline Mexico & 0 & 0 & 0 & 0 & 1 & 1 & 0 & 0 & 0 & 0 & 0 & 0 & 1 & 1 \\
\hline Nicaragua & 0 & 0 & 0 & 1 & 0 & 0 & 0 & 0 & 0 & 0 & 0 & 0 & 0 & 1 \\
\hline Panama & 0 & 0 & 0 & 1 & 0 & 0 & 0 & 0 & 0 & 0 & 0 & 0 & 0 & 1 \\
\hline Central America, unspecified & 0 & 1 & 1 & 5 & 0 & 0 & 0 & 0 & 0 & 0 & 0 & 3 & 1 & 9 \\
\hline South America & 1 & 1 & 9 & 25 & 1 & 1 & 0 & 0 & 0 & 0 & 1 & 10 & 12 & 37 \\
\hline Brazil & 0 & 0 & 2 & 5 & 0 & 0 & 0 & 0 & 0 & 0 & 0 & 2 & 2 & 7 \\
\hline Colombia & 1 & 1 & 0 & 1 & 1 & 1 & 0 & 0 & 0 & 0 & 0 & 1 & 2 & 4 \\
\hline Guyana & 0 & 0 & 4 & 7 & 0 & 0 & 0 & 0 & 0 & 0 & 0 & 4 & 4 & 11 \\
\hline Peru & 0 & 0 & 3 & 9 & 0 & 0 & 0 & 0 & 0 & 0 & 1 & 3 & 4 & 12 \\
\hline Venezuela & 0 & 0 & 0 & 3 & 0 & 0 & 0 & 0 & 0 & 0 & 0 & 0 & 0 & 3 \\
\hline Oceania & 0 & 1 & 2 & 4 & 0 & 0 & 0 & 0 & 0 & 0 & 0 & 1 & 2 & 6 \\
\hline Papua New Guinea & 0 & 1 & 2 & 4 & 0 & 0 & 0 & 0 & 0 & 0 & 0 & 1 & 2 & 6 \\
\hline Unknown & 4 & 22 & 0 & 3 & 0 & 0 & 0 & 2 & 0 & 0 & 3 & 13 & 7 & 40 \\
\hline Total & 497 & 1,409 & 90 & 248 & 62 & 96 & 25 & 61 & 20 & 23 & 17 & 219 & 711 & 2,056 \\
\hline
\end{tabular}

Abbreviation: $\mathrm{PCR}=$ polymerase chain reaction.

patients provided a reason for travel; two patients traveled for missionary work, and one each for business or education (as a student or teacher).

Confirmed cases were classified according to location of diagnosis or residence; all 50 states reported at least one case of malaria. Fifteen jurisdictions reported $>50$ cases of malaria in 2016, accounting for $74.6 \%$ of the 2,078 cases reported: New York City (265), Maryland (182), Texas (170), California (125), Massachusetts (95), New Jersey (86), Pennsylvania (84), Florida (79), Virginia (75), New York state (not including New York City) (74), Georgia (69), Minnesota (66), Illinois (65), Ohio (63), and North Carolina (52) (Figure 2). Thirty-seven jurisdictions reported an increase in cases, and 16 reported no change or a decrease in cases. Five jurisdictions reported $\geq 35$ more cases in 2016 compared with 2015: Texas (70 more), Maryland (55 more), New York City (47 more) Pennsylvania (37 more), and Massachusetts (35 more). Seven jurisdictions reported $>20$ more cases in 2016 compared with 2015: Arizona, Ohio, and Michigan (26 more), North Carolina (25 more), Minnesota (23 more), Washington and Florida (21 more).
Rhode Island had the most substantial decrease in malaria cases in 2016 compared with 2015, with seven fewer cases reported.

\section{Imported Malaria Among U.S. Residents and Nonresidents}

Among 2,056 imported cases, information on residence status was available for 1,834 (89.2\%) cases; of these, 1,253 $(68.3 \%)$ were U.S. residents, and 581 (31.7\%) were non-U.S. residents (Table 4). The majority of infections among U.S. residents were acquired in Africa (1,087 [86.8\%]), which was comparable to $2015(826[86.5 \%])$ and continues a trend that has occurred since 2008 (11,36,39-44). Among U.S. residents, an increase occurred in the number of imported cases acquired in Asia (47 [5.0\%] in 2015 versus 88 [7.1\%] in 2016). A decrease occurred from 2015 to 2016 among U.S. residents who acquired malaria from the Caribbean (36 [3.8\%] in 2015 versus 28 [2.3\%] in 2016). No significant change occurred from 2015 to 2016 in the number of U.S. residents with imported malaria infections from Central America (17 
FIGURE 2. Number* of malaria cases, by state — United States, 2016

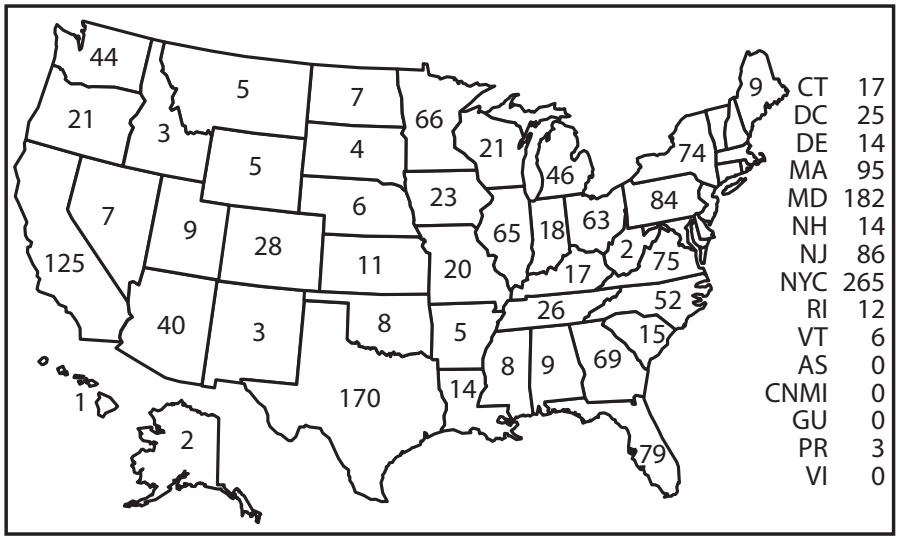

Abbreviations: $\mathrm{AS}=$ American Samoa; $\mathrm{CT}=$ Connecticut $\mathrm{CNMI}=$ Commonwealth of the Northern Mariana Islands; $D C=$ Washington, $D C ; D E=$ Delaware; $\mathrm{GU}=$ Guam; $\mathrm{MA}=$ Massachusetts; $\mathrm{MD}=$ Maryland; $\mathrm{NH}=$ New Hampshire; $\mathrm{NJ}=$ New Jersey; NYC = New York City; PR = Puerto Rico; RI = Rhode Island; $\mathrm{VI}=$ Virgin Islands; $\mathrm{VT}=$ Vermont.

${ }^{*} \mathrm{~N}=2,078$. New York state cases do not include those from New York City.

[1.8\%] in 2015 versus 11 [0.9\%] in 2016) or from South America (18 [1.9\%] in 2015 versus 23 [1.9\%] in 2016). The number of cases acquired among U.S. residents from Oceania remained $<1.0 \%$ in 2015 and 2016 (2 [0.2\%] in 2015 versus four [0.3\%] in 2016).

The majority of malaria cases imported among non-U.S. residents in 2016 were from Africa (474 [81.6\%]), followed by Asia (70 [12.1\%]) and the Americas and Caribbean (35 [6.0\%]) (Table 4). Among non-U.S. residents, a significant increase occurred in the numbers of imported malaria cases from Africa in 2016 compared with 2015 (274 cases [74.5\%] in 2015 versus 474 cases [81.6\%] in 2016). From Asia, although the number of cases among non-U.S. residents increased in 2016 (from 64 cases in 2015, to 70 cases in 2016), the proportion significantly decreased in 2016 (18.1\% in 2015 versus $12.1 \%$ in 2016 ). Altogether, the number of non-U.S. residents with malaria who traveled from South and Central America and the Caribbean region to the United States increased in 2016 compared with 2015; 35 cases (36.5\%) in 2016 compared with 15 cases (17.4\%) in 2015. From 2015 to $2016, \geq 20$ more cases were reported among residents of Nigeria (67 cases in 2015 and 126 cases in 2016), Democratic Republic of the Congo (15 cases in 2015, 50 cases in 2016), Uganda (12 cases in 2015 and 40 cases in 2016), Tanzania (nine cases in 2015 and 34 cases in 2016), and Cameroon (17 cases in 2015 and 37 cases in 2016).

The decrease from 2014 to 2015 in the proportion of U.S. residents who acquired malaria from West Africa and Ebolaaffected countries coincided with the Ebola outbreak in West Africa and associated travel warnings (45-47). From 2015 to 2016, the proportion of U.S residents who acquired malaria in West Africa was not significantly different (542 [56.8\%] in
TABLE 4. Number and percentage of imported malaria cases among U.S. residents and non-U.S. residents, by region of acquisition United States, 2016

\begin{tabular}{|c|c|c|c|c|c|c|}
\hline \multirow[b]{2}{*}{ Area or region } & \multicolumn{2}{|c|}{ U.S. residents } & \multicolumn{2}{|c|}{ Non-U.S. residents } & \multicolumn{2}{|c|}{ Total } \\
\hline & No. & (\%) & No. & (\%) & No. & (\%) \\
\hline Africa & 1,087 & (86.8) & 474 & (81.6) & 1,561 & (85.1) \\
\hline Asia & 88 & (7.0) & 70 & $(12.1)$ & 158 & (8.6) \\
\hline South America & 23 & (1.8) & 11 & (1.9) & 34 & (1.9) \\
\hline $\begin{array}{l}\text { Central America } \\
\text { and the } \\
\text { Caribbean }\end{array}$ & 39 & (3.1) & 24 & (4.1) & 63 & (3.4) \\
\hline Oceania & 4 & $(0.3)$ & 0 & (0) & 4 & $(0.2)$ \\
\hline Unknown & 12 & (1.0) & 2 & $(0.3)$ & 14 & $(0.8)$ \\
\hline Total & 1,253 & (100) & 581 & (100) & 1,834 & (100) \\
\hline
\end{tabular}

2015 versus 739 [59.0\%] in 2016). However, from 2015 to 2016 the proportion of U.S. residents who acquired malaria from previously Ebola-affected countries increased significantly (224 [17.8\%] in 2016 versus 91 [9.5\%] in 2015). Among nonU.S. residents, the number of cases acquired in West Africa increased from 2015 to 2016 (175 in 2015, 227 in 2016), although the proportion significantly decreased $(47.6 \%$ in 2015, 39.1\% in 2016). From 2015 to 2016, the proportion of cases among non-U.S. residents that acquired malaria from Ebola-affected countries decreased significantly (41 [11.4\%] in 2015 versus 42 [7.2\%] in 2016).

\section{Seasonality of Malaria Diagnosed in the United States}

As in previous years $(11,36,42-44)$, imported malaria cases peaked during the summer holiday months of July and August 2016, with a mean of 161.3 imported cases across all months and an average of 271.5 cases in July and August 2016 (Figure 3). The fewest numbers of malaria cases were reported in February and March 2016, with 74 cases each. In 2016, a total of 554 more cases were reported than in 2015, and the numbers of cases reported in every month of 2016 were greater than in the same month of 2015. The average percent increase from 2015 to 2016 was $39.8 \%$ across all months. May had the largest percent increase $(67.3 \%)$ and November the smallest increase $(7.7 \%$ increase). $P$. falciparum species accounted for $380(70.0 \%)$ of 543 cases imported in July and August 2016, and $80(54.1 \%)$ of 148 cases in February and March 2016. $P$. vivax and $P$. ovale infections were combined because the illnesses can relapse, and a secondary peak can occur after the main peak. In 2016, the mean number of $P$. vivax and $P$. ovale cases was 27.6 per month; the maximum numbers occurred in July and August (45 and 48 cases, respectively). The numbers of $P$. vivax and $P$. ovale cases were greater than the mean during May-October. P. vivax and P. ovale cases were high in May 2016, suggesting a potential secondary peak 
FIGURE 3. Number* of imported malaria cases, by Plasmodium species and month of symptom onset - United States, 2016

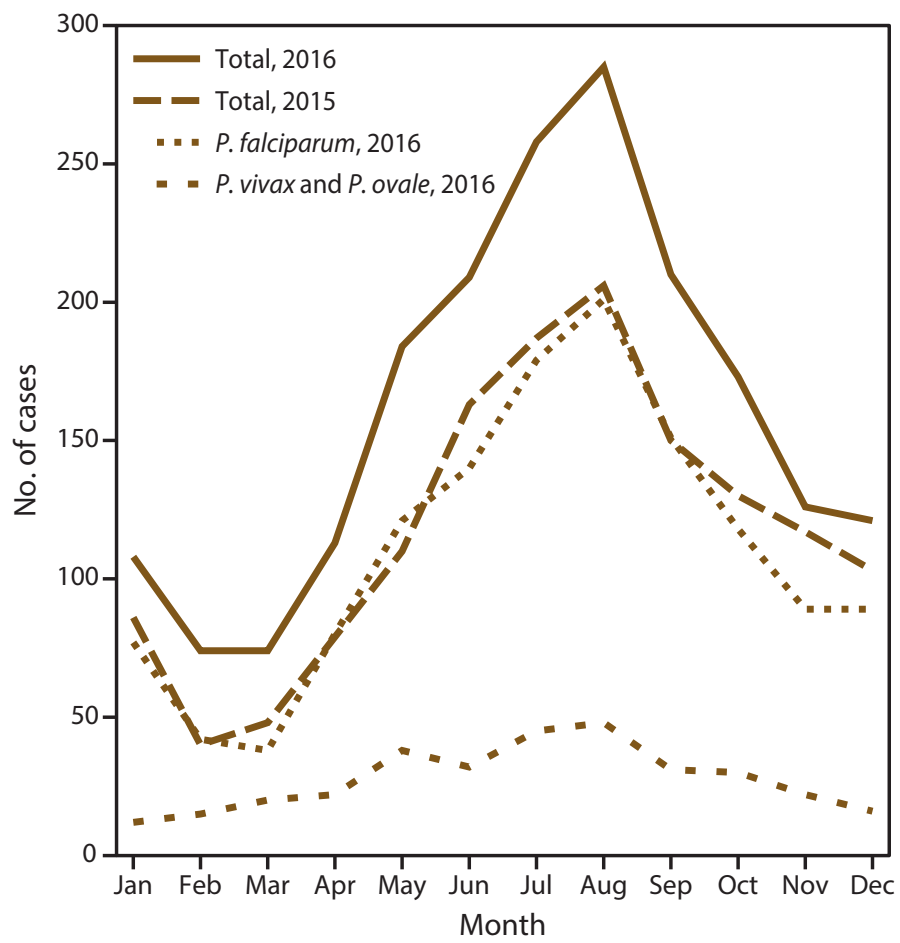

* Total number of cases for 2016 was 1,935, which includes the following: 279 P. malariae, mixed, and unknown species determination; 1,325 infections with $P$. falciparum; and 331 infections with $P$. vivax and $P$. ovale. Total number of cases for 2015 was 1,419 infections with all species.

from relapsing infections, which typically occurs $>45$ days after exposure, often among patients reporting a previous history of malaria (Figure 3). During January-June, an average of 4.5 P. vivax cases per month were categorized as probable acute, which increased to 16.0 cases per month during the summer months of July-September and decreased to an average of 4.6 cases per month during October-December. In contrast, the $P$. vivax cases categorized as probable relapses showed a wider seasonal distribution, with a peak of 24 cases in May, and ranged from 21 to 24 cases during May-August. The proportion of probable acute $P$. vivax cases that occurred during peak months (July-September) was 67 of 130 cases (51.5\%), significantly higher than the proportion of probable relapse cases, with 28 of 106 cases (26.4\%) occurring during peak months. Although the numbers of cases were lower, seasonality patterns were not found among the $P$. ovale probable relapse or probable acute cases (Figure 4).

\section{Interval Between Arrival in the United States and IIIness Onset}

Among 1,837 imported cases with a Plasmodium species determination, 1,443 (78.6\%) had complete information on
FIGURE 4. Number* of Plasmodium vivax and Plasmodium ovale imported malaria cases, by month of symptom onset and probable relapse — United States, 2016

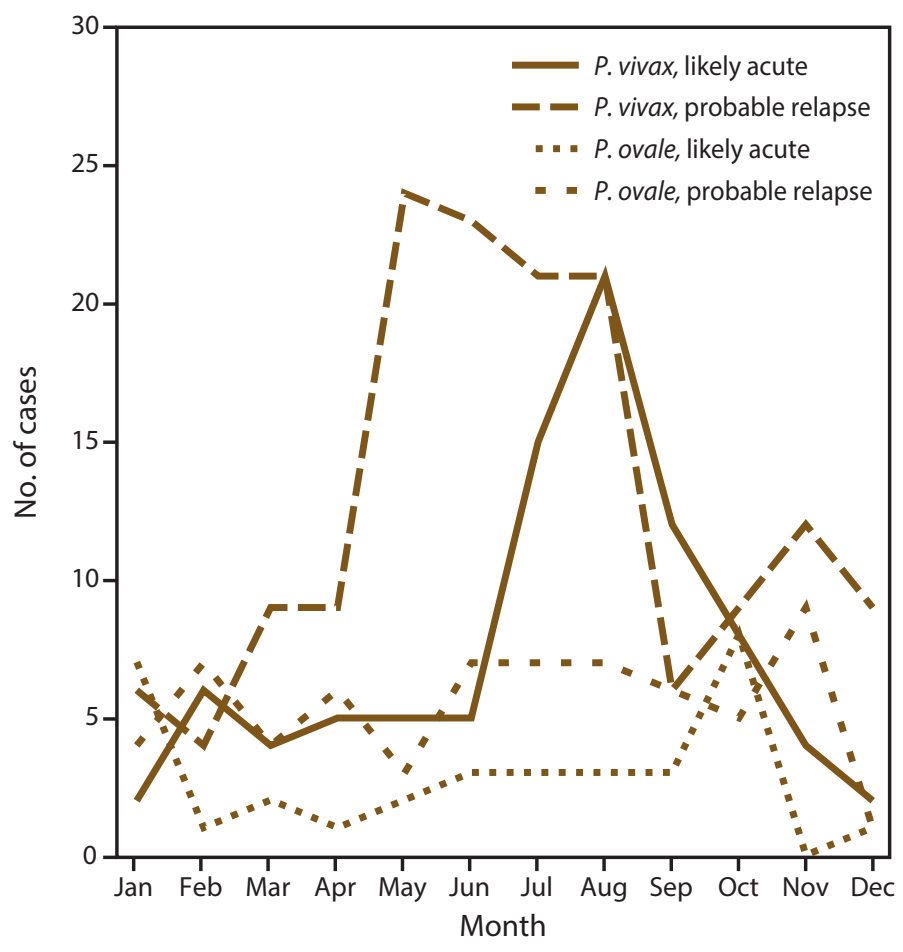

* Across all months, the number of likely $P$. vivax acute infections was 89 and probable $P$. vivax relapse infections was 153 . The number of likely $P$. ovale acute infections was 34 and probable $P$. ovale relapse infections was 66 .

return travel and illness onset dates, allowing for calculation of the interval between dates (Table 5). Among all patients, regardless of infecting species, $172(11.9 \%)$ had symptom onset before arriving in the United States. Among 1,144 patients with P. falciparum infections, 1,074 (93.9\%) had symptom onset before or within 29 days of arrival to the United States. In contrast, 87 (49.7\%) of 175 P. vivax patients and 32 (46.4\%) of $69 P$. ovale patients had illness onset $\geq 30$ days after arrival in the United States, consistent with the potential for these species to relapse because of the persistence of liver hypnozoites. Ninety-nine percent of infections with any species occurred within 1 year of arrival in the United States after travel to a country where malaria is endemic.

\section{Imported Malaria Among U.S. Military Personnel}

In 2016, a total of 41 cases of malaria diagnosed in the United States occurred among U.S. military personnel, compared with 23 cases reported in 2015 (1.5\% and $2.0 \%$ of imported cases in 2015 and 2016, respectively). In 2016, a total of 17 cases of malaria among U.S. military members were acquired in Africa, 17 cases were acquired in Asia, one was acquired from 
TABLE 5. Number and percentage of imported malaria cases, by Plasmodium species and interval between date of arrival in the United States and onset of illness* — United States, 2016

\begin{tabular}{|c|c|c|c|c|c|c|}
\hline & P. falciparum & P. vivax & P. ovale & P. malariae & Mixed & Total \\
\hline Interval (days) & No. (\%) & No. (\%) & No. (\%) & No. (\%) & No. (\%) & No. (\%) \\
\hline$<0^{\dagger}$ & 147 (12.9) & $12(6.9)$ & $5(7.3)$ & $6(14.6)$ & $2(14.3)$ & 172 (11.9) \\
\hline $0-29$ & $927(81.0)$ & $76(43.4)$ & $32(46.4)$ & $19(46.3)$ & $6(42.9)$ & $1060(73.5)$ \\
\hline $30-89$ & $53(4.6)$ & $44(25.1)$ & $18(26.1)$ & $10(24.4)$ & $6(42.9)$ & $131(9.1)$ \\
\hline $90-179$ & $9(0.8)$ & $24(13.7)$ & $7(10.1)$ & $4(9.8)$ & $0(0)$ & $44(3.1)$ \\
\hline $180-364$ & $5(0.4)$ & $18(10.3)$ & $6(8.7)$ & $1(2.4)$ & $0(0)$ & $30(2.1)$ \\
\hline$\geq 365$ & $3(0.3)$ & $1(0.6)$ & $1(1.5)$ & $1(2.4)$ & $0(0)$ & $6(0.4)$ \\
\hline Total & $1,144(100.0)$ & $175(100.0)$ & $69(100.0)$ & $41(100.0)$ & $14(100.0)$ & $1,443(100.0)$ \\
\hline
\end{tabular}

* Persons for whom Plasmodium species, date of arrival in the United States, or date of onset of illness is unknown are not included.

† Cases in this row are in patients who had onset of illness before arriving in the United States.

the Caribbean, and the country or region of acquisition was unknown for six cases. Among the cases acquired in Africa, nine cases were acquired among service personnel in Middle Africa (Cameroon [seven], Democratic Republic of Congo [one], and Gabon [one]), five were acquired in West Africa (Niger [one], Nigeria [one], Sierra Leone [one], Togo [one], and West Africa unspecified [one]), and three were from Africa (country unspecified). Four of the five cases acquired in West Africa were acquired during personal leave, including one case from Sierra Leone. The number of military cases acquired in West Africa was lower in 2016 than in 2015 (nine [45.0\%] cases in 2015 versus five [14.3\%] cases in 2016). The number of military cases acquired from Asia was higher in 2016 than in 2015 (17 [48.6\%] in 2016 versus four [20.0\%] in 2015). Of the 17 military cases acquired in Asia in 2016, 10 were from South Korea and seven from Afghanistan. Military cases from Afghanistan and South Korea increased from 2015 to 2016, from two cases to seven cases and two cases to 10 cases, respectively. The military personnel with cases acquired from Asia had a significantly longer interval between date of return to the United States and symptom onset (mean interval: 113.4 days, range: $3-415$ days) compared with cases acquired in Africa (mean interval: 11.0 days, minimum: $-4-46$ days). The Plasmodium species was known for 36 of 41 military cases diagnosed in the United States in 2016. Of these, 20 (55.6\%) were $P$. falciparum, 15 (41.7\%) were $P$. vivax, and one $(2.8 \%)$ was $P$. malariae. Nine $(60.0 \%)$ of the 15 patients who were infected with $P$. vivax, received primaquine to treat liver hypnozoites.

Thirty (73.2\%) military patients had information on chemoprophylaxis use; 10 did not take an antimalarial medication to prevent malaria. Of the 20 patients who took chemoprophylaxis, 11 took an appropriate regimen for the region of travel; nine took doxycycline, one took mefloquine, and one patient took two or more medications for chemoprophylaxis. Eight of eleven patients with chemoprophylaxis information reported missing doses. Nine
(45\%) of 20 patients did not report chemoprophylaxis regimen information. Of the seven military patients who acquired malaria from Cameroon, information on chemoprophylaxis was available for six, and all six reported taking some chemoprophylaxis. Regimen information was available on four of the six who took chemoprophylaxis, and all four took doxycycline; five of six reported missing doses (and one patient had no adherence information reported). Of the seven cases that were acquired in Afghanistan, five patients reported taking chemoprophylaxis for prevention (two reported not taking chemoprophylaxis). Doxycycline was taken by three of the five patients who had traveled to Afghanistan, and all of them reported missing doses of the chemoprophylaxis medication. Of the 10 military patients who traveled to South Korea, only one reported taking chemoprophylaxis, and no details on the regimen or adherence was reported. No uniform policy exists requiring active-duty military personnel to take chemoprophylaxis during deployment in many parts of South Korea $(5,48)$.

Three military patients received a diagnosis of and treatment for severe malaria in the United States. All three patients recovered.

\section{Chemoprophylaxis Use Among U.S. Residents}

Among U.S. residents, information about malaria chemoprophylaxis use was reported for $1,103(88.0 \%)$ of 1,253 imported cases; among these patients, 812 (73.7\%) indicated that no chemoprophylaxis regimen was taken during travel. Among the 291 patients who reported taking chemoprophylaxis, $90(30.9 \%)$ did not indicate which medication was taken. Among 201 patients who reported specific drug information, $157(78.1 \%)$ took a regimen recommended by CDC for the region of travel. Among 132 reports containing self-reported adherence information, 74 (56.1\%) patients reported missing doses. The U.S. residents 
with malaria who initiated an appropriate chemoprophylaxis regimen but did not adhere to the entire regimen (74 of 157 [47.1\%]) provided reasons for missing doses, which included prematurely stopping after leaving the area where malaria was endemic (23.0\%), forgetting to take the medication (20.3\%), and stopping due to a side effect (13.5\%); unknown and other reasons (e.g., lost medication, inaccurate prescriptions, and child unable to swallow the medication) also were reported, although less frequently. Among patients who took chemoprophylaxis, reported adherence was higher in 2016 than in 2015 (58 [43.9\%] of 132 in 2016 versus 37 [28.9\%] of 128 in 2015). Among 157 patients who took CDC-recommended chemoprophylaxis, 64 (40.8\%) took doxycycline, 55 (35.0\%) took mefloquine, 31 (19.8\%) took atovaquone-proguanil, and seven $(4.5 \%)$ took two or more CDC-recommended medications. Among the 964 U.S. residents with complete information on chemoprophylaxis, $58(6.0 \%)$ patients were adherent to an appropriate regimen, $832(86.3 \%)$ patients did not take chemoprophylaxis or took an incorrect regimen, and $74(7.7 \%)$ patients did not adhere to an appropriate regimen. Ninety-four percent (906 of 964) of U.S. residents with malaria did not adhere to or did not take a CDC-recommended chemoprophylaxis regimen.

\section{Cases of $P$. vivax or $P$. ovale Infections After CDC- Recommended Prophylaxis Use}

The infecting malaria species was known for 139 (88.5\%) of 157 patients who took a CDC-recommended chemoprophylaxis regimen; P. vivax accounted for 18 (13.0\%) and P. ovale for 13 $(9.4 \%)$ of these cases. Primary prophylaxis can prevent acute illness in $P$. vivax and $P$. ovale infections, although the patient might experience a relapsing illness unless primaquine is taken to eliminate dormant hypnozoites (radical cure) (24). A previous history of malaria or illness onset $>45$ days after return to the United States are conditions that are consistent with relapsing illnesses. Three of $31 P$. vivax and $P$. ovale patients who took chemoprophylaxis reported a previous history of malaria within the past 12 months. Sufficient information was available for 23 of these $31 P$. vivax or $P$. ovale patients to calculate the number of days between the return travel date and the date of illness onset; symptom onset occurred $>45$ days after return to the United States among 14 patients. Nine cases occurred $\leq 45$ days after return to the United States, and the patients reported no history of previous illness, suggesting an acute infection and possible primary prophylaxis failure; eight of nine patients did not adhere to the chemoprophylaxis regimen, and one patient did not provide adherence information. Of the nine $P$. vivax or $P$. ovale patients who took some chemoprophylaxis, seven took doxycycline and two took mefloquine.

\section{P. falciparum, P. malariae, or Mixed Infections After CDC-Recommended Prophylaxis Use}

Among the 139 patients with known Plasmodium species and who reported taking a CDC-recommended prophylaxis regimen, 101 (72.7\%) of the infections were from P. falciparum, and seven $(5.0 \%)$ were from $P$. malariae. Among the patients with $P$. falciparum infections, $99(98.0 \%)$ reported travel to Africa, and $62(61.4 \%)$ had traveled to West Africa. One $P$. falciparum case each occurred after persons traveled to Asia (country unspecified) and Papua New Guinea. Of 88 P. falciparum case reports containing information on adherence to the recommended prophylaxis regimen, 47 (53.4\%) patients indicated nonadherence. Thirty-seven cases of P. falciparum occurred among patients who reported adherence to a recommended chemoprophylaxis regimen; 13 (35.1\%) patients took mefloquine, 12 (32.4\%) took doxycycline, 10 (27.0) took atovaquone-proguanil, and two (5.4\%) took two or more antimalarial regimens for chemoprophylaxis. All 37 patients with $P$. falciparum infections who adhered to chemoprophylaxis acquired their infections in Africa, with $26(70.3 \%)$ from the West Africa region. CDC received nine specimens for molecular resistance surveillance from patients who adhered to chemoprophylaxis, four patients took atovaquone-proguanil, three took mefloquine, and two took doxycycline (one took another medication not recommended for chemoprophylaxis in the United States, in addition to doxycycline) for chemoprophylaxis. Of the four patients who took only atovaquone-proguanil, all had traveled to West Africa (Guinea, Ghana, Togo, and Nigeria), and none had mutations in genes known to cause resistance to atovaquone, suggesting that known resistance mutations were not a factor. Of the three who reported adherence to mefloquine for prophylaxis, two were siblings who traveled together to Uganda, and one patient traveled to Ghana. One of the siblings had a $p f m d r 1$ copy number amplification, which is known to be associated with mefloquine resistance. Valid genetic markers are not available to assess doxycycline resistance.

Seven cases of $P$. malariae occurred after patients had taken a recommended prophylaxis regimen. All seven patients had information on adherence; two patients reported taking all doses of chemoprophylaxis, and five did not adhere to the regimen. Specimens were not available for CDC testing for the two patients that reported adherence. One patient traveled to Ghana for 3 months, took doxycycline, and received confirmation of $P$. malariae infection by blood smear. The second patient traveled to Zambia for 24 days, took atovaquone-proguanil for chemoprophylaxis, and had PCR confirmation of $P$. malariae infection. Possible explanations for Plasmodium infections in patients who adhered to chemoprophylaxis include inadequate 
dosing or malabsorption of the chemoprophylaxis medication, inaccurate reporting of adherence, emerging parasite resistance, or chemoprophylaxis failure.

\section{Patients with a Recent History of Malaria}

Among the 2,056 cases of malaria imported into the United States in 2016, a total of $1,523(74.1 \%)$ patients provided information on previous history of malaria; 356 (23.4\%) reported having had malaria in the past 12 months. The infecting species for the previous illness was reported for 42 (11.8\%) of 356 patients with a previous history of malaria; 21 (50.0\%) recalled $P$. falciparum, $16(38.1 \%)$ recalled $P$. vivax, one $(2.4 \%)$ recalled $P$. ovale, three $(7.1 \%)$ recalled $P$. malariae, and one $(2.4 \%)$ recalled a mixed infection with $P$. falciparum and $P$. ovale. On the basis of illness onset date, previous date of illness, and species, 13 probable relapse cases were identified, all caused by $P$. vivax. Four additional cases were considered to be probable relapses (three $P$. vivax and one $P$. ovale) because the species was the same for the previous and current illnesses; however, the previous date of illness onset was missing. Among the 21 patients who recalled a $P$. falciparum infection in the past 12 months, 19 (90.5\%) had a current infection with P. falciparum. Among the three patients who recalled a previous P. malariae infection in the past 12 months, two were infected with species other than P. malariae, and one was infected with an unspecified species.

\section{Reason for Travel}

Among nonmilitary U.S. residents, the reason for travel to a country where malaria is endemic was reported for 987 (81.4\%) of 1,212 imported malaria cases (Table 6). Of these, $714(72.3 \%)$ case reports included VFR as the reason for travel; missionary travel was reported in $87(8.1 \%)$, and business travel was reported in 69 (7.0\%), a decrease from 2015 (74

TABLE 6. Number* and percentage of imported malaria cases among U.S. civilians and non-U.S. residents, by reason for travel at the time of acquisition - United States, 2016

\begin{tabular}{lrrr}
\hline & U.S. civilians & & Non-U.S. residents \\
\cline { 2 - 2 } Category & No. (\%) & & No. (\%) \\
\hline Visiting friends and relatives & $714(58.9)$ & & $118(20.3)$ \\
Tourist & $74(6.1)$ & & $15(2.6)$ \\
Missionary or dependent & $87(7.2)$ & & $2(0.3)$ \\
Business & $69(5.7)$ & & $15(2.6)$ \\
Student or teacher & $23(1.9)$ & & $40(6.9)$ \\
Air crew or sailor & $3(0.3)$ & & $1(0.7)$ \\
Peace Corps & $3(0.3)$ & & $0(0)$ \\
Refugee or immigrant & $0(0)$ & & $263(45.3)$ \\
Other & $14(1.4)$ & & $20(3.4)$ \\
Unknown & $225(18.6)$ & & $107(18.4)$ \\
Total & $1,212(100)$ & & $581(100)$ \\
\hline
\end{tabular}

${ }^{*} \mathrm{~N}=1,793$ cases $[9.8 \%])$. Tourism was reported by $74(7.5 \%)$ patients. Twenty-three $(2.3 \%)$ patients acquired malaria during travel for education (as students or teachers). Three $(0.3 \%)$ cases were related to Peace Corps service in 2016. Three (0.3\%) cases occurred among airline or ship crew members. Fourteen patients provided other reasons for travel (1.5\%). In 2016, no difference occurred in the number of U.S. residents that contracted malaria after traveling to Africa or Asia for tourism. However, from 2015 to 2016, the number of U.S. resident tourists who contracted malaria from South and Central America and the Caribbean in 2016 decreased, with 15 (24.2\%) cases in 2016 compared with 30 (42.3\%) in 2015.

Among the 581 imported cases among non-U.S. residents, reason for travel was available for $474(81.6 \%)$ cases (Table 6). Among those who provided a reason for travel, traveling to the United States as an immigrant or a refugee was cited in 263 case reports (55.5\%), an increase from 2015 (121 [44.6\%]). VFR travel to the United States from countries where malaria is endemic was reported by 118 (24.9\%) patients, followed by 15 (3.2\%) patients reporting business travel, a decrease compared with 2015 (19 [7.0\%]). Forty (8.4\%) patients traveled for education (as a student or teacher) in 2016. Tourism was reported by $15(3.2 \%)$ patients, missionary travel was reported by two $(<1.0 \%)$ patients, and airline or ship crew travel was reported by one $(<1.0 \%)$ patient. Other reasons were reported by $20(4.2 \%)$ patients. Of the 263 non-U.S. residents who traveled to the United States as a refugee or an immigrant, 215 $(81.8 \%)$ originated in Africa, followed by Asia (35 [13.3\%]), and the Americas and Caribbean (12 [4.6\%]); one patient who was a refugee or immigrant did not report a country of origin.

\section{Malaria by Age}

Age was reported for all 2,078 of the patients with malaria in 2016, and of these, adults aged $\geq 18$ years accounted for $1,713(82.4 \%)$ cases. A total of $104(5.0 \%)$ patients were aged $<5$ years, and $130(6.3 \%)$ were aged $\geq 65$ years. Of the 365 children (aged $<18$ years), $118(32.3 \%)$ were U.S. residents, and 107 (90.7\%) of these had traveled to Africa. Among the U.S. civilian children, 81 (68.6\%) were VFR travelers, $11(9.3 \%)$ traveled for missionary reasons, five $(4.2 \%)$ for tourism, and three $(2.5 \%)$ for education. No reason for travel was provided for $15(14.6 \%)$ children, and two provided other reasons for travel. Among the 118 U.S. civilian children, information on chemoprophylaxis use and adherence was reported for 105 (89.0\%); 59 (56.2\%) did not take medication during travel to prevent malaria. Of the 46 children who took a chemoprophylaxis regimen, 26 (56.5\%) took a CDC-recommended antimalarial that was appropriate for the region of travel. Of the 22 children with information 
on adherence, case reports from eight (33.3\%) indicated that all doses were taken. All eight cases were acquired in Africa; six cases were confirmed $P$. falciparum, one case was severe, and no deaths were reported.

\section{Hospitalization}

In 2016, hospitalization information was reported for 1,831 (88.1\%) of 2,078 confirmed cases; of these, 1,261 (68.9\%) patients were hospitalized for their malaria illness. A total of $918(72.8 \%)$ hospitalized patients had $P$. falciparum malaria in 2016, and 268 (21.3\%) hospitalized patients had one or more signs or symptoms of severe malaria. No significant change in these percentages occurred from 2015 to 2016; in 2015, 985 $(72.0 \%)$ patients were hospitalized among 1,368 with this information reported, $714(72.5 \%)$ with $P$. falciparum, 236 $(24.0 \%)$ with severe malaria (not mutually exclusive).

\section{Treatment for Uncomplicated Malaria Cases}

\section{Overall}

Among the 2,078 confirmed malaria cases in 2016, a total of $1,772(85.3 \%)$ cases were uncomplicated. Of these, $1,513(85.4 \%)$ had antimalarial treatment information indicated on the case report form. The most common treatment administered for uncomplicated malaria in 2016 was atovaquone-proguanil, used to treat 879 (58.1\%) of imported cases in patients with treatment information. Quinine-based treatment ranked second, with 247 (16.3\%) patients treated with this regimen. Artemether-lumefantrine, the only artemisinin-based combination therapy approved by the Food and Drug Administration (FDA) for treatment of uncomplicated malaria in the United States, was used for 184 $(12.2 \%)$ patients. A total of 154 (10.2\%) uncomplicated cases were treated with chloroquine, and $82(5.4 \%)$ were treated with mefloquine.

The CDC Guidelines for Treatment of Malaria in the United States, herein referred to as the CDC guidelines, provides guidance for the treatment of malaria according to species, disease severity, and region of acquisition (22). Of the 1,513 imported uncomplicated cases with treatment information, 1,365 (90.2\%) were treated in accordance with CDC guidelines, a similar proportion to the number of uncomplicated cases treated correctly in 2015 (926 [90.1\%]). Of those treated correctly in 2016, 180 (13.2\%) patients reported taking an additional antimalarial medication that exceeded what is recommended by the CDC guidelines. Among the $103(10.0 \%)$ patients whose treatment was not in accordance with CDC recommendations, seven (4.7\%) were treated with the same antimalarial that the patient had used for chemoprophylaxis. To avoid potential toxicity and reduced efficacy, patients should not be treated with the same antimalarial that was used for chemoprophylaxis.

\section{Adequacy of Treatment by Species}

Among the 1,012 uncomplicated P. falciparum cases, 928 (91.7\%) were treated according to CDC guidelines, and 132 $(14.2 \%)$ of these patients received an antimalarial treatment that exceeded the recommendations. Six $(7.1 \%)$ of 84 P. falciparum patients who were not treated in accordance with CDC recommendations were pregnant. Treatment followed CDC guidelines for 49 (90.7\%) of 54 cases of uncomplicated P. malariae, and eight (16.3\%) of those patients received an additional antimalarial medication. Of those with $P$. vivax and P. ovale infections, $178(87.7 \%)$ and 75 (92.6\%), respectively, were treated following CDC guidelines for their acute infection, of whom nine $(5.1 \%)$ patients with $P$. vivax infections and eight $(16.3 \%)$ with $P$. ovale infections received an additional acutephase antimalarial medication. Administration of primaquine to treat relapsing illness (the liver hypnozoite stage of the parasite) was reported for 91 (45.5\%) of all 200 nonpregnant patients with $P$. vivax infections and treatment information. Among the 79 nonpregnant patients with $P$. ovale infections, primaquine was administered to $32(40.5 \%)$. Fourteen $(77.8 \%)$ of 18 mixed infections with treatment information recorded were treated according to CDC guidelines; one (7.1\%) of these patients received an additional antimalarial. Malaria infections with an unknown species should be treated following recommendations for $P$. falciparum (22); 121 (83.5\%) of 145 cases with unknown infecting species were treated according to recommendations, and $22(18.2 \%)$ of these were treated with an additional antimalarial for the acute illness. Of 144 nonpregnant patients with unknown infecting species, 18 $(12.5 \%)$ were treated with primaquine.

\section{Severe Malaria}

In 2016, a total of 306 (14.7\%) of 2,078 cases were classified as severe malaria. Seven $(2.3 \%)$ of the patients with severe malaria died. Among severe cases, 240 (78.4\%) occurred in adults aged 18-64 years. However, patients with severe malaria were more likely to be children than adults ( 66 of 365 [18.1\%] children aged $<18$ years versus 240 of 1,713 [14.0\%] adults aged $\geq 18$ years). The proportion of adults aged $\geq 65$ years with severe malaria was $19.2 \%$ ( 25 of 130 cases), whereas the proportion of severe malaria among patients aged $<65$ years was $14.4 \%$ ( 281 of 1,948 cases). In 2016, patients with severe malaria were equally likely to be U.S residents or non-U.S. residents; a total of 196 (15.6\%) of 1,257 U.S. residents, compared with $93(16.0 \%)$ of 581 non-U.S. residents had 
severe malaria. Among patients with severe malaria in 2016, $261(85.3 \%)$ had $P$. falciparum infections, 22 (7.2\%) had infections with an unknown Plasmodium species, and 15 (4.9\%) patients had P. vivax infections. Approximately $\leq 1 \%$ of severe infections were due to $P$. malariae $(\mathrm{n}=2)$, $P$. ovale $(\mathrm{n}=2)$, or mixed species infections $(\mathrm{n}=4)$. In 2016, the species distribution among severe cases was similar to the distribution in 2015.

Among 268 patients with severe malaria and information on chemoprophylaxis (87.6\% of 306 severe cases), 45 (16.8\%) reported having taken any medication for chemoprophylaxis; 24 (53.3\%) of these did not provide adherence or chemoprophylaxis medication information. Among 21 patients with severe malaria and known chemoprophylaxis adherence information, $15(71.4 \%)$ did not adhere to or take a recommended regimen for malaria prevention, compared with $58.6 \%$ of persons with uncomplicated cases who did not adhere to chemoprophylaxis or take a correct regimen. Of the six patients who adhered to a recommended chemoprophylaxis regimen, five took doxycycline and one took atovaquone-proguanil. Two specimens from these six patients were tested at CDC for molecular resistance markers, including from one patient who took atovaquone-proguanil; the specimen from this patient did not contain markers of known resistance to atovaquone. No validated markers for doxycycline resistance are available to evaluate the specimen from the patient with severe malaria who adhered to doxycycline for chemoprophylaxis. Potential reasons for chemoprophylaxis failure in these patients with severe malaria include inadequate dosing or malabsorption of the chemoprophylaxis medication, inaccurate reporting of adherence, or emerging parasite resistance.

Patients with severe malaria can have multiple clinical complications; acute kidney injury was most common and was experienced by $56(18.3 \%)$ patients. Cerebral malaria was reported for $33(10.8 \%)$ patients, severe anemia for 35 (11.4\%), acute respiratory distress syndrome for 18 (5.9\%), and jaundice for 12 (3.9\%). Case reports from 245 patients with severe malaria contained information on the percentage of Plasmodium species parasitemia, of which 168 (68.6\%) had $\geq 5 \%$ parasitized red blood cells. CDC guidelines in 2016 stated that patients with severe malaria should be treated aggressively in an inpatient setting with intravenous quinidine gluconate or artesunate. In 2016, a total of 19 (6.2\%) patients with severe malaria were not hospitalized; the hospitalization status for another 19 (6.2\%) patients was unknown. At that time, the only FDA-approved medication for this indication was intravenous quinidine gluconate; for patients who could not tolerate quinidine gluconate or when quinidine was not available, artesunate could be obtained through CDC through an investigational new drug protocol (49). In 2016, a total of
138 (45.1\%) patients with severe malaria were treated with quinidine gluconate, 29 (9.5\%) were treated with artesunate, and $18(5.9 \%)$ were treated with both quinidine gluconate and artesunate. A total of 185 (60.5\%) patients with severe malaria were treated with a parenteral antimalarial regimen, and $109(35.6 \%)$ were treated with an oral regimen alone; treatment information was unknown for 12 (3.9\%) patients. Among seven patients who died, one received both quinidine gluconate and artesunate, one received quinidine gluconate alone, one received artesunate alone, and two received an oral antimalarial treatment; two patients received no antimalarial treatment because they did not seek care and malaria was diagnosed after they were found unresponsive or dead. Among 50 pregnant women, 17 had severe malaria, of whom 12 received parenteral treatment: eight received quinidine gluconate, two received artesunate, and two received both parenteral treatments. Five pregnant women with severe malaria received oral antimalarial treatment.

In 2016, a total of 147 (49.7\%) of 296 patients with severe malaria and information on treatments received appropriate treatment, a significant reduction compared with 2015, when $146(58.9 \%)$ of 248 patients received appropriate treatment. In addition, in 2016, patients with severe malaria were significantly more likely to have inappropriate treatment (149 [50.2\%] of 296) than patients with uncomplicated malaria (148 [9.8\%] of 1,513). Although all malaria Plasmodium species can cause severe malaria, the rapid proliferation of $P$. falciparum parasites in nonimmune persons requires timely diagnosis and treatment to prevent progression to hyperparasitemia and severe illness. In 2016, on average, inpatients with $P$. falciparum infections were hospitalized 4.9 days after illness onset, regardless of disease severity (with a mean of 4.2 days for uncomplicated cases and 5.4 days for severe cases).

Among the 306 patients with severe illness, 285 (93.1\%) infections were acquired from Africa; eight (2.6\%) from Asia; seven $(2.3 \%)$ from the Caribbean; and $<1.0 \%$ from Oceania (two cases), Central America (one case), and South America (one case). Two severe cases were acquired in an unknown region. Among the 250 patients with severe malaria who reported a reason for travel, $135(54.0 \%)$ reported VFR as the primary reason travel, of whom $126(93.3 \%)$ had acquired the infections in Africa, and 95 (75.4\%) of these from West Africa. Forty-seven $(12.4 \%)$ patients with severe malaria traveled to the United States as immigrants or refugees, 22 (8.8\%) patients traveled for tourism, and 18 (7.2\%) traveled for business. Eight patients $(3.2 \%)$ with severe malaria traveled for education (as a student or teacher), and three were members of the U.S. military. Two patients with severe malaria had other reasons for travel, and reason for travel was unknown for 56 (18.3\%). 


\section{Malaria During Pregnancy}

Among 795 women with malaria diagnosed in the United States in 2016, 50 (6.3\%) were pregnant, and 17 (34.0\%) of these had severe malaria. Forty-one $(82.0 \%)$ pregnant women were hospitalized, including 15 patients with severe malaria, and all recovered. Data on birth outcomes are not systematically collected.

Thirty-seven $(74.0 \%)$ pregnant women had $P$. falciparum infections, three $(6.0 \%)$ each were infected with $P$. vivax, $P$. malariae, and an unspecified Plasmodium species. Two (4.0\%) women each had P. ovale and mixed species infections. Over half of the pregnant women (28 [56.0\%]) were nonU.S. residents, and 17 (34.0\%) women were U.S. residents; residence status was not provided for five $(10.0 \%)$ women. Of $38(76.0 \%)$ women who indicated a reason for travel, 18 (47.4\%) were VFR travelers, 14 (36.8\%) were refugees or immigrants, two (5.3\%) traveled for tourism, and one each $(4.0 \%)$ traveled as a missionary, for education (as a student or a teacher), as a member of an airline or ship crew, or for another reason.

Antimalarial drug choices to prevent or treat malaria during pregnancy are limited. In most areas where malaria is endemic, only mefloquine is approved for chemoprophylaxis; in 2016, either mefloquine or quinine with clindamycin was recommended as treatment for uncomplicated malaria during pregnancy (22). Pregnant women can use chloroquine for chemoprophylaxis or for the treatment of uncomplicated malaria acquired from areas with chloroquine-sensitive malaria. Primaquine can cause hemolytic anemia among persons with glucose-6-phosphate dehydrogenase (G6PD) deficiency and should not be administered during pregnancy. Severe malaria in pregnant women should be treated aggressively with intravenous regimens; in 2016, the options were parenteral quinidine-gluconate or artesunate.

Sixteen (94.1\%) of 17 pregnant women who were U.S. residents provided information on chemoprophylaxis use; 15 women did not take chemoprophylaxis to prevent malaria during travel, and one took mefloquine in accordance to the CDC guidelines for prevention of malaria among pregnant women. The woman who took mefloquine for chemoprophylaxis reported adherence to the regimen. She traveled for 2 months to visit friends and relatives in Cameroon and was diagnosed with uncomplicated $P$. falciparum infection (1.2\% parasitemia); specimens were not examined for molecular resistance markers.

Treatment information was available for 46 (92.0\%) pregnant women with malaria. Of the 29 patients with uncomplicated malaria and information on antimalarial treatments, 17 (58.6\%) were treated appropriately for malaria.
Three $(10.3 \%)$ patients received atovaquone-proguanil, which is not recommended for use during pregnancy. Three $(10.3 \%)$ pregnant women with uncomplicated malaria were treated with artemether-lumefantrine, which at the time of treatment in 2016 was not recommended for use in pregnant women but subsequently became endorsed for use in pregnant women in 2018 (50). One (2.0\%) pregnant woman with an uncomplicated $P$. falciparum infection was improperly treated with primaquine in 2016; primaquine is contraindicated for pregnancy and should not be administered for $P$. falciparum infections.

Of the 17 pregnant women with severe malaria, 14 (82.4\%) were infected with $P$. falciparum, one (5.9\%) each was infected with $P$. malariae, multiple species, and an unknown species. Among pregnant women with severe malaria, seven (41.2\%) were treated inappropriately; five $(29.4 \%)$ pregnant patients with severe malaria used oral treatment only.

Among 32 pregnant women with P. falciparum infections and complete data, two $(6.3 \%)$ were considered late recurrences, with symptom onset starting $>45$ days after travel. This is similar to the number from 2015, with two late recurrences among 20 (10.0\%) P. falciparum infections.

\section{Drug Resistance Markers}

In 2016, CDC received 222 whole blood samples from patients with malaria. Of these, 63 samples were from patients who did not have $P$. falciparum infections, and samples from six patients did not amplify any markers of resistance; therefore, no resistance monitoring was conducted. Molecular resistance surveillance was performed on $144 P$. falciparum samples and nine mixed infection samples (four with $P$. falciparum and $P$. vivax, two each with $P$. falciparum plus $P$. ovale or $P$. falciparum plus $P$. malariae, and one triple infection with P. falciparum, P. ovale, and P. malariae) (Table 7). However, not all loci amplified for each of the markers analyzed. Of the 145 P. falciparum samples that amplified for pyrimethamine resistance, 142 (97.9\%) contained polymorphisms associated with pyrimethamine resistance, and 132 (93.0\%) of these had three or more pyrimethamine resistance markers. A total of 139 samples amplified for sulfadoxine resistance analysis, and 98 $(70.5 \%)$ had at least one marker associated with sulfadoxine resistance; nine $(9.2 \%)$ of these had three or more sulfadoxine resistance markers. Of 150 samples tested for chloroquine resistance markers, $67(44.7 \%)$ had a resistant genotype. Amplification of the mefloquine locus was completed for 141 samples, and six (4.3\%) contained the resistant genotype. The genes associated with atovaquone resistance were amplified for 147 samples, and one $(<1.0 \%)$ sample each had one or two polymorphisms associated with resistance. One of the 
Surveillance Summaries

TABLE 7. Antimalarial drug resistance marker results among Plasmodium falciparum specimens, by drug and region of malaria acquisition -United States, 2016

\begin{tabular}{|c|c|c|c|c|c|c|c|}
\hline \multirow[b]{3}{*}{ Resistance markers } & \multicolumn{6}{|c|}{ Area or region } & \multirow[b]{2}{*}{ Total } \\
\hline & Africa & Asia & $\begin{array}{c}\text { Central America } \\
\text { and the Caribbean }\end{array}$ & $\begin{array}{c}\text { South } \\
\text { America }\end{array}$ & Oceania & Unknown & \\
\hline & No. (\%) & No. (\%) & No. (\%) & No. (\%) & No. (\%) & No. (\%) & No. (\%) \\
\hline Pyrimethamine & $138(95.2)$ & $0(0)$ & $2(1.4)$ & $0(0)$ & $0(0)$ & $5(3.5)$ & $145(100)$ \\
\hline No resistance markers & $2(1.4)$ & $0(0)$ & $1(0.7)$ & $0(0)$ & $0(0)$ & $0(0)$ & $3(2.1)$ \\
\hline 1 resistance marker & $0(0)$ & $0(0)$ & $1(0.7)$ & $0(0)$ & $0(0)$ & $0(0)$ & $1(0.7)$ \\
\hline 2 resistance markers & $9(6.2)$ & $0(0)$ & $0(0)$ & $0(0)$ & $0(0)$ & $0(0)$ & $9(6.2)$ \\
\hline$\geq 3$ resistance markers & 127 (87.6) & $0(0)$ & $0(0)$ & $0(0)$ & $0(0)$ & $5(3.5)$ & $132(91.0)$ \\
\hline Sulfadoxine & (132) 95.0 & $0(0)$ & $2(1.4)$ & $0(0)$ & $0(0)$ & $5(3.6)$ & $139(100)$ \\
\hline No resistance markers & $40(28.8)$ & $0(0)$ & $0(0)$ & $0(0)$ & $0(0)$ & $1(0.7)$ & $41(29.5)$ \\
\hline 1 resistance marker & $64(46.0)$ & $0(0)$ & $2(1.4)$ & $0(0)$ & $0(0)$ & $3(2.2)$ & 69 (49.6) \\
\hline 2 resistance markers & $19(13.7)$ & $0(0)$ & $0(0)$ & $0(0)$ & $0(0)$ & $1(0.7)$ & $20(14.4)$ \\
\hline$\geq 3$ resistance markers & $9(6.5)$ & $0(0)$ & $0(0)$ & $0(0)$ & $0(0)$ & $0(0)$ & $9(6.5)$ \\
\hline Chloroquine & $142(94.7)$ & $1(0.7)$ & $2(1.3)$ & $0(0)$ & $0(0)$ & $5(3.3)$ & $150(100)$ \\
\hline No resistance markers & $76(50.7)$ & $0(0)$ & $2(1.3)$ & $0(0)$ & $0(0)$ & $5(3.3)$ & $83(55.3)$ \\
\hline 1 or 2 resistance markers & $0(0)$ & $0(0)$ & $0(0)$ & $0(0)$ & $0(0)$ & $0(0)$ & $0(0)$ \\
\hline 3 resistance markers & $66(44.0)$ & $1(0.7)$ & $0(0)$ & $0(0)$ & $0(0)$ & $0(0)$ & $67(44.7)$ \\
\hline Mefloquine & $134(95.0)$ & $0(0)$ & $2(1.4)$ & $0(0)$ & $0(0)$ & $5(3.6)$ & $141(100)$ \\
\hline No resistance markers & $128(90.8)$ & $0(0)$ & $2(1.4)$ & $0(0)$ & $0(0)$ & $5(3.6)$ & $135(95.7)$ \\
\hline 1 resistance marker & $6(4.3)$ & $0(0)$ & $0(0)$ & $0(0)$ & $0(0)$ & $0(0)$ & $6(4.3)$ \\
\hline Atovaquone & $139(94.6)$ & $1(0.7)$ & $2(1.4)$ & $0(0)$ & $0(0)$ & $5(3.4)$ & $147(100)$ \\
\hline No resistance markers & $137(93.2)$ & $1(0.7)$ & $2(1.4)$ & $0(0)$ & $0(0)$ & $5(3.4)$ & $145(98.6)$ \\
\hline 1 resistance marker & $1(0.7)$ & $0(0)$ & $0(0)$ & $0(0)$ & $0(0)$ & $0(0)$ & $1(0.7)$ \\
\hline 2 resistance markers & $1(0.7)$ & $0(0)$ & $0(0)$ & $0(0)$ & $0(0)$ & $0(0)$ & $1(0.7)$ \\
\hline Artemisinin & $146(95.4)$ & $0(0)$ & $2(1.3)$ & $0(0)$ & $0(0)$ & $5(3.3)$ & $153(100)$ \\
\hline No polymorphism & $145(94.8)$ & $0(0)$ & $2(1.3)$ & $0(0)$ & $0(0)$ & $5(3.3)$ & $152(99.4)$ \\
\hline A578S polymorphism* & $1(0.7)$ & $0(0)$ & $0(0)$ & $0(0)$ & $0(0)$ & $0(0)$ & $1(0.7)$ \\
\hline
\end{tabular}

* Specimen observed with the A578S polymorphism that is not associated with antimalarial resistance.

153 samples investigated for artemisinin resistance had the 578S K13 mutation commonly found in Africa.

Because of widespread resistance to pyrimethamine and sulfadoxine, CDC does not recommend using drugs containing these components to treat malaria in the United States (22). None of the patients with chloroquine resistance markers had exposure to malaria in a chloroquine-sensitive region, and $76(91.6 \%)$ of the chloroquine-sensitive samples were from patients who acquired malaria in Africa. All six patients with markers of mefloquine resistance had traveled to Africa (Uganda) and five of them to West Africa (Sierra Leone [two patients], Togo [two patients], and Ghana). Of these six, three reported taking chemoprophylaxis, two took mefloquine (of whom one reported complete adherence while the second reported missing doses). The patient who did not adhere to the mefloquine chemoprophylaxis had severe malaria with $6 \%$ P. falciparum infection. The two illnesses in these patients might have resulted from induced mefloquine resistance. No patients with a mefloquine resistance genotype were treated with mefloquine, and all recovered from their illness. The two patients who acquired malaria with atovaquone resistance had traveled to Burkina Faso and did not take chemoprophylaxis; one patient (aged 29 years) had two markers of atovaquone resistance, and one patient (aged 1 year) had one marker of atovaquone resistance. Both patients experienced a recrudescence of parasitemia after treatment with atovaquoneproguanil, which is presumed to have resulted from induced parasite resistance. Subsequent to atovaquone-proguanil treatment failure, the patient aged 29 years was treated with quinine plus doxycycline, and the patient aged 1 year was treated with quinidine-gluconate and clindamycin. Both patients recovered from their illness. CDC testing detected the A578S K13 polymorphism commonly found in Africa but not associated with artemisinin resistance (34) in a sample from one patient who had traveled to Guinea and took no prophylaxis.

\section{Selected Malaria Case Reports}

\section{Congenital Cases}

Two cases of congenital malaria were reported in 2016. Parasites were transmitted from mother to child during pregnancy or during labor and delivery.

Case 1. A black, pregnant woman aged 22 years emigrated from Liberia to the United States in October 2016. Two days after arrival, she sought care at the emergency department at 40.5 weeks gestation (estimated by ultrasound), experiencing abdominal cramping and jaundice. She received a diagnosis 
of $P$. falciparum malaria, with $<1 \%$ parasitemia and was hospitalized. At the time of admission she had severe anemia (hemoglobin: 6.5, hematocrit: 18.7) and jaundice. She was treated for severe anemia with the transfusion of 6 units of blood. A male infant was born by caesarian section 4 days after the mother was admitted. After birth, the infant had hypoglycemia and a poor appetite, and a blood smear was positive for $P$. falciparum (with no percentage parasitemia reported). Both the mother and infant were treated with oral quinine and clindamycin, and both recovered. The infant was discharged 8 days after birth.

Case 2. Despite requests for additional information, limited details were available for this patient (an infant) and her mother, an Asian woman. In 2015, approximately 5 months before the birth, the mother immigrated to the United States from Afghanistan. The mother had a history of malaria illness, and approximately 9 months before the infant's birth, she received 3 days of antimalarial treatment while in Afghanistan; no additional details on the mother's illness or recovery are available, and the mother did not receive a diagnosis of or treatment for malaria after arriving in the United States. The female infant was born in 2016 in the United States; within a month of the birth, she was febrile and received a diagnosis of P. vivax malaria and anemia, with $1.6 \%$ parasitemia. The infant was treated for malaria and recovered from her illness, although the type of antimalarial medication used was not reported.

\section{Transfusion-Transmitted Malaria}

In 2016, one case of malaria transmitted through a blood transfusion in the United States was reported, the first since 2011 (11).

Recipient. A black man aged 18 years with sickle cell anemia was admitted to the hospital in July 2016 for pain and fever related to a sickle cell crisis; the man had not traveled to a country where malaria is endemic and had received multiple transfusions in June 2016. His fever continued, and he experienced encephalopathy; 2 days after admission, he was transferred to the intensive care unit (ICU), where he continued to have spiking fevers. He experienced multiorgan failure, underwent intubation and mechanical ventilation, and received additional blood transfusions, with an exchange transfusion 2 days after transfer to the ICU. The following day (5 days after admission), the hospital identified parasites by blood smear examination. The state public health laboratory examined the blood smear and initially reported the species as P. vivax. Subsequently, PCR was performed and the species was changed to $P$. falciparum, with $4 \%$ parasitemia according to a blood smear performed after the exchange transfusion. After aggressive treatment with intravenous artesunate, the recipient recovered but required rehabilitation in a long-term care facility.
Investigation. The recipient received blood products from four donors in June 2016, and samples from all four were tested by CDC via immunofluorescence (IFA) assay. One of the four samples was positive (1:1024) for P. falciparum. The blood transfusions during the July hospitalization (after symptom onset) did not initiate the malaria illness. The state health department attempted to contact the IFA-positive donor through numerous calls and mailed letters but were unsuccessful. In December 2016, the health department visited the donor's home and were able to conduct an interview and collect whole blood samples for additional testing. The whole blood sample collected from the donor in December 2016 was PCR positive for $P$. falciparum.

Donor. The donor was a woman (race not reported) aged approximately 37 years at the time of her donation in June 2016. She was born in the United States but lived in the Democratic Republic of Congo when aged 3-20 years. During that time, she reported that she had malaria every year or so, with the last illness at age 19 or 20 years. Since moving to the United States, she had been working for an airline and traveled extensively to destinations often in Africa (especially Democratic Republic of Congo, Burundi, and Rwanda). She provided her passports to the state health department to document her extensive travel history. Her last trip to a country where malaria was endemic had been a 2-week trip to Democratic Republic of Congo in February 2015. She reported that she did not become ill or febrile after that trip, nor was she ill or febrile at the time of the donation. She reported that she had not had malaria since she left Democratic Republic of Congo at age 20 years. She had been deemed eligible to donate blood because $>1$ year had elapsed since her last travel to a country where malaria was endemic, and during the past 16 years she had not lived for $>5$ years in a country where malaria was endemic. The donor had no other significant medical history, although she gave birth in November 2015. In December 2016, the donor was asymptomatic, despite testing positive for malaria by PCR. The state health department informed her of the test results and referred her for antimalarial treatment.

\section{Cryptic Cases}

Three cases were classified as cryptic because the patients had not traveled recently, no relapsing malaria, no health care or blood exposure (e.g. transfusion, needle stick, nosocomial infection, intravenous drug use, or home tattooing), and a low likelihood of local transmission.

Cases 1 and 2. Adult Hispanic siblings (a sister aged 27 years and her brother aged 22 years) had been living in the southeastern United States in a six-person household. Both patients had been born in Honduras and reported no international travel since their arrival in the United States. The 
woman had immigrated to the United States 7 years earlier, and the man had arrived $2^{1 / 2}$ years before onset of his illness.

The sister was pregnant and gave birth to a healthy child in October 2016. The woman's fever began 4 days after childbirth; 9 days after onset of the fever, she was hospitalized. P. falciparum malaria was diagnosed 3 days later, with a parasitemia of $0.05 \%$. She was treated with atovaquoneproguanil and recovered.

The brother's fever onset occurred 6 days after the onset for the sister, and he was admitted to the hospital 1 day after she was admitted. He received a diagnosis of uncomplicated P. falciparum malaria on the same day as his sister. No percentage parasitemia was reported. He was treated with atovaquone-proguanil and doxycycline and recovered.

During the course of the investigation, the siblings were interviewed by their health care providers, state and local health departments (on two occasions), and a visiting nurse. The interviews did not reveal any other likely exposure (e.g., intravenous drug use or blood exposure, contact with a sick visitor, or a connection with another case in the area). The sister shared her passport, which showed no recent travel. Her medical records showed that she had attended seven prenatal medical visits with local providers during March-October 2016.

Two days after the cases were diagnosed, entomological surveillance using seven mosquito traps was conducted as part of the investigation by the state and local health departments. All traps were incandescent or ultraviolet CDC light traps. Two ultraviolet light traps were set 0.52 and 0.9 miles from the siblings' residence for four nights; one incandescent light trap and one ultraviolet light trap were set within 0.08 miles of the siblings for two nights; one incandescent light trap was set 0.19 miles away for one night; and two ultraviolet light traps were set for two nights within 0.24 miles of the residence. No Anopheles mosquitos were caught during the investigation trapping. As part of routine surveillance, eight Anopheles mosquitos were collected during October 11-18, 2016, at a site 2.9 miles from the residence. The county health department sprayed for mosquitos on October 25.

Whole blood samples from the siblings were tested by PCR at the $\mathrm{CDC}$ reference laboratory; molecular resistance markers and microsatellite profiles were examined. The $P$. falciparum parasite signatures from both patients were consistent with the profile of parasites originating from Central America. Microsatellite analysis showed that the sister was infected with two separates strains of $P$. falciparum; her brother was infected with one strain, which was identical to one of the strains causing illness in his sister. Because of local climatological conditions in mid-October 2016, the chances of continued malaria transmission in this area would have been very low.
Case 3. A black woman aged 48 years who lived in the northeastern United States sought care from her primary care physician in August 2016 after experiencing fever, weakness, and fatigue for 1 day. A blood smear was sent to a clinical laboratory, and 2 days later the results indicated that the patient was infected with $P$. falciparum malaria with $0.5 \%$ parasitemia. The smear was not forwarded to a public health laboratory for confirmation. The patient was treated with mefloquine and was asymptomatic when interviewed 10 days later. She had been born in Liberia but reported that she had not traveled in the last 4 years, which was confirmed by a passport inquiry. She reported no recent history of malaria infection or health care exposure risk factors (e.g., blood transfusions or receipt of donated organs or tissues). No contact with ill persons was reported; whether her other household members had traveled to countries where malaria is endemic before her illness onset is unknown. Mosquito surveillance data for the 2016 season were examined to assess the possibility of local malaria transmission. The only identification of Anopheles species mosquitos in the woman's zip code occurred 3 days before her illness onset at a location 0.8 miles away from her home (six A. quadrimaculatus mosquitos).

\section{Fatal Cases}

Seven fatal malaria cases were reported in 2016.

Case 1. A white man aged 50 years spent 1 month in Equatorial Guinea for work and returned to the United States in January 2016; his chemoprophylaxis use was unknown. He experienced a diarrheal illness the day he returned, which persisted for 2 weeks. He reported no fever during this time. When he noticed darkening of his urine, accompanied by decreased urine output and urinary urgency during the second week of February, he sought medical care. He was admitted to the hospital with a temperature of $103^{\circ} \mathrm{F}\left(39.4^{\circ} \mathrm{C}\right)$, severe jaundice, and acute kidney injury. One day after admission, he provided his travel history, and atovaquone-proguanil was started empirically while a blood smear malaria test was ordered. The blood smear results were reported the following day, indicating $P$. falciparum malaria infection, with an estimated 7\%-20\% parasitemia. He was treated with intravenous quinidine gluconate and doxycycline. The man experienced refractory hypotension, which made dialysis difficult. Although the parasitemia resolved by his fourth day in the hospital, the man died 3 days later.

Case 2. An Asian man aged 45 years traveled to India for 1 month and returned to the United States in August 2015. His chemoprophylaxis use was unknown. Approximately 9 months later, in April 2016, he experienced fever and headache and sought care from his primary care physician the next day. At the time, he received a diagnosis of a respiratory infection, 
was given antibiotics, and was sent home. Three days later, his family found him unresponsive at home and called an ambulance. When the ambulance and emergency medical services (EMS) personnel arrived, he had an altered mental status and was combative; he was given lorazepam (Ativan). When he arrived at the hospital, his breathing was agonal, and he was exhibiting decerebrate posturing. Advanced cardiac life support was initiated but unsuccessful, and the man was pronounced dead 40 minutes later. Blood samples taken during the resuscitation efforts showed incidental intracellular organisms in the manual differential. The CDC reference laboratory confirmed $P$. vivax infection on a subsequent blood smear. Cause of death was severe $P$. vivax malaria.

Case 3. A black woman aged 62 years traveled to Nigeria to visit friends and relatives and returned to the United States in June 2016. The duration of her travel and whether she took chemoprophylaxis is unknown. She experienced fever and influenza-like symptoms 2 days after her return to the United States. After 2-3 days of symptoms, she called an ambulance and was seen by EMS personnel, who told her she had influenza and did not need emergent medical attention. One week later she was found unresponsive, and EMS personnel were called again. The first responders found a bottle of nortriptyline under her; EMS personnel administered naloxone and transported her via ambulance to the emergency department. En route, the woman experienced asystole, and cardiopulmonary resuscitation was initiated. When she arrived at the hospital, she was still unresponsive and in asystole. Cardiopulmonary resuscitation was continued, and the woman was pronounced dead within an hour of arrival. An autopsy was performed, and a postmortem blood specimen revealed presence of $P$. falciparum parasite, with $10 \%$ parasitemia. The medical examiner determined cerebral malaria to be the cause of death.

Case 4. A black man aged 52 years worked and visited friends and family for 10 months in Liberia during 2015-2016; he did not use malaria chemoprophylaxis. While still in Liberia, 3 weeks before his eventual admission to the hospital in the United States, he noticed the onset of increasing abdominal girth and bilateral lower extremity edema. He reported no fever. In July 2016, he returned to the United States and sought medical attention. His initial assessment indicated that he had no fever but had jaundice, thrombocytopenia, and acidosis with acute kidney injury. An abdominal ultrasound indicated hepatic cirrhosis and portal hypertension. The man was admitted to the hospital for evaluation and management of the newly diagnosed liver disease. Soon after admission, he experienced a cardiac arrest. He underwent intubation and mechanical ventilation, and was resuscitated and transferred to the ICU. The hematology laboratory identified malaria parasites on his complete blood count slide, which were subsequently identified as $P$. falciparum, with $5.8 \%$ parasitemia. Intravenous quinidine was ordered but before it could be administered, the man experienced another cardiac arrest and died 1 day after admission to the hospital.

Case 5. In July 2016, a Hispanic woman aged 54 years who lived in Guyana arrived in the United States to visit friends and relatives. Nine days after arrival, she experienced fever, chills, and a headache. Her symptoms persisted, and 3 days later she sought medical attention at an emergency department. She was febrile with thrombocytopenia. Malaria parasites were observed on the blood smear, and she was admitted with a diagnosis of $P$. vivax malaria, which was later confirmed by PCR at the regional reference laboratory. She was treated initially with 4 doses of oral quinine and doxycycline, which was switched to chloroquine. Her G6PD tests were normal, and primaquine was administered. Forty-eight hours after admission, she developed acute respiratory distress syndrome requiring intubation and mechanical ventilation. Malaria smears 5, 10, and 11 days after hospitalization were all negative. On the morning of her 11 th day in the hospital, she experienced acute hypoxemia and bradycardia. Although she was resuscitated, she remained hypoxemic and died later that day.

Case 6. In August 2016, a black woman aged 41 years returned to the United States from Burkina Faso, where she had been visiting family and friends for 2 weeks; she did not take chemoprophylaxis. A fever began the day after her return, and she was admitted to the hospital 3 days later with severe P. falciparum malaria with a reported hyperparasitemia of $50 \%$, lactic acidosis, and jaundice. Because the hospital had no quinidine in stock and was unable to obtain any, health care providers contacted CDC to obtain artesunate. During the evening, the day after hospital admission, she received her first dose of artesunate. After her initial evaluation, she began developing shortness of breath requiring supplemental oxygen. Her shortness of breath progressed to severe acute respiratory distress syndrome requiring intubation and mechanical ventilation with $100 \%$ oxygen. Her respiratory status did not improve. Although she received all 4 doses of artesunate and the parasitemia decreased to $5.2 \%$, she experienced severe hypoxemia and died after 4 days in the hospital. After her death, a whole blood sample was sent to CDC for diagnostic confirmation, and PCR revealed that she had a mixed species infection with $P$. falciparum and $P$. vivax.

Case 7. A black man aged 24 years had recently arrived in the United States after travel to Guinea, where he did not take chemoprophylaxis. He received a diagnosis of severe cerebral malaria in October 2016. His initial parasitemia was estimated to be $15 \%-20 \%$ with $P$. falciparum, and he had a normal neurologic exam. Soon after admission he had a seizure and 
underwent intubation and mechanical ventilation. An initial head computed tomography (CT) scan appeared normal. He received intravenous quinidine. After he experienced QTc prolongation, the dosage was adjusted. CDC was contacted, and artesunate was sent for use if the cardiac toxicity persisted. In the morning, 2 days after hospitalization, the man's pupils were fixed and nonreactive. A repeat head CT scan showed cerebral edema with herniation. Parasitemia had decreased to $0.2 \%$. Intravenous artesunate was administered. After 4 doses of artesunate he did not recover, and ventilatory support was withdrawn. He died 4 days after admission to the hospital.

\section{Discussion}

A record 2,078 malaria cases were diagnosed in the United States in 2016, an increase from 2015 when 1,524 malaria cases were diagnosed. During 2010-2014, an average of 1,754 cases per year were reported. In 2015, significantly fewer cases were acquired from West Africa than in 2014, although no other significant regional decreases occurred. The decreased number of malaria cases in 2015 was likely due to the Ebola outbreak.

The United Nations World Tourism Organization (UNWTO) reported tourist arrival data for two of three Ebola-affected countries. From 2014 to 2015, tourist arrivals across Africa decreased by $2.9 \%$, and although arrivals in Guinea increased by $6.1 \%$, they decreased in Sierra Leone by 45.6\% (51). More recently, UNWTO reported that travel across Africa was 7.8\% higher in 2016 than in 2015, with approximately 58 million international tourists. Travel to the two Ebola-affected countries was higher in 2016 than in 2015; arrivals to Guinea increased by $71.4 \%$ and to Sierra Leone by $125.7 \%$. Travel to Southeast Asia increased by $9 \%$ in 2016, and travel to India increased by $10 \%$. In 2016, despite Zika virus transmission, tourist arrivals to South America increased by $7 \%$ and increased to Central America by $5 \%(51,52)$.

The number of imported malaria cases has been increasing since the mid-1970s, with the highest number of cases since 1972 reported in 2016 (Table 1). This peak of malaria cases might be explained by global travel trends to and from areas where malaria is endemic. Although the overall number of cases has increased, the proportion of cases from the regions of acquisition has remained stable, with one recent exception; in 2015, a transient decrease in the number of cases acquired from West Africa (36). This brief change might be attributable to decreased travel to these Ebola-affected areas for a limited time (36). In 2016, the number of malaria cases increased across most regions of the world with malaria transmission.

The proportion of P. falciparum infections (68.3\%) in 2016 is comparable to that in 2015 (67.3\%); these percentages are the highest recorded for the period 2005-2016. Use of PCR testing to identify the Plasmodium species has also improved, which might have contributed to this finding. Likewise, the proportion of undetermined species is the lowest reported during 2012-2016 (10.8\% in 2016; mean proportion of species undetermined from 2012 to $2015: 14.5 \%)$. Travel to the African region also might have contributed to the large proportion of $P$. falciparum infections; UNWTO reported an overall increase in travel rates to sub-Saharan Africa, with 39.2 million international tourist arrivals reported for 2016, an increase from 35.4 million in 2015 (51). Likewise, flights from the United States to Africa among U.S. citizens increased by approximately 12,300 per year during 1996-2015 (36). From 2015 to 2016, the outbound flights from the United States to Africa increased by 14,320 (37). An analysis of the GeoSentinel global surveillance network, which monitors travel-related illness, indicated that $83 \%$ of travelers who contracted malaria were exposed in sub-Saharan Africa (53). Likewise, a meta-analysis with data from 2005-2016 showed that among imported malaria infections in 40 countries where malaria is not endemic, $56 \%$ of cases were from West Africa (54).

During 2015-2016, the Zika virus spread to South and Central America and the Caribbean (55,56). In early 2016, public health officials announced that Zika virus was a public health emergency of international concern because of its ability to cause microcephaly and other birth defects $(57,58)$. Beginning in January 2016, CDC began issuing travel health notices for countries experiencing Zika virus transmission, especially pertaining to pregnant women and their partners and to persons trying to conceive (59). Efforts to improve awareness of Zika virus risk in 2016 included mosquito avoidance measures, which might have been protective against malaria (60). Although the number of malaria cases imported from South America, Central America, and the Caribbean did not change significantly from 2015 to 2016, a decrease occurred in the number of U.S. resident tourists who contracted malaria. Moreover, the number of non-U.S. residents with malaria who traveled from this region to the United States increased from 2015 to 2016. One pregnant malaria patient traveled to the United States from this region (Central America, country unspecified).

Studies among refugees from Africa to North America showed that a high proportion of these persons are infected with malaria, predominantly $P$. falciparum $(61,62)$. Asymptomatic parasitemia is common among persons who lived in areas with $P$. falciparum transmission $(63,64)$. Consequently, since 2012, as part of the U.S. refugee health examination, refugees originating from sub-Saharan Africa are administered directly observed artemether-lumefantrine as presumptive treatment for malaria before departure to the United States (38). In 
2016, children weighing < $11 \mathrm{lb}(5 \mathrm{~kg})$ and pregnant women were not given presumptive treatment but instead were tested for malaria and received appropriate treatment if infected. Refugees originating from other regions of the world are assessed for fever during the refugee health examination and are tested for malaria if symptomatic.

In early 2017, refugee health coordinators diagnosed cases of $P$. malariae among refugees who relocated to the United States from sub-Saharan Africa and notified CDC. Typically, the observed $P$. malariae cases were detected in persons of Congolese nationality, often in refugee camps in countries situated in central and eastern Africa: Tanzania, Uganda, and the Central African Republic. A retrospective analysis of the NMSS surveillance data presented in this report suggest that although the overall proportion of the $P$. malariae species did not change significantly from 2015 to 2016, an increase occurred in $P$. malariae cases among Congolese refugees or refugees from the indicated countries, from $6.3 \%$ in 2015 to $31.2 \%$ in 2016. Limitations of this analysis are that only eight of 19 P. malariae cases in 2016 were PCR confirmed. In addition, classification errors are more likely with P. malariae species because of its microscopic characteristics, which resemble $P$. falciparum; furthermore, based on the species name, case report form submitters are more likely to erroneously select $P$. malariae as the species instead of selecting Plasmodium species unspecified. CDC attempts to correct classification errors with health department and case report form submitters, nonetheless, errors might persist. To assist in future surveillance monitoring, in February 2017, CDC notified state health departments about this observation and emphasized the importance of PCR confirmation, especially for cases suspected of $P$. malariae species, and among refugees from sub-Saharan Africa. Although artemether-lumefantrine was assessed to be an effective treatment for $P$. malariae and other non-P. falciparum species (65), persistence of parasitemia has been observed in patients with $P$. malariae infections $(7,8,66)$. One hypothesis is that because of the slow preerythrocytic development period in the liver, this parasite might be more likely to evade erythrocyte-targeted treatments than other species (67).

The first case of transfusion-transmitted malaria in the United States since 2011 occurred in 2016 (11). Unfortunately, 5 days passed from when the recipient first developed fever until receipt of a malaria diagnosis, and the already ill patient experienced severe malaria and endured an extended recovery process. The United States does not have a licensed test to screen donors for malaria to protect the blood supply; therefore, FDA recommends that donor history questionnaires be used to determine eligibility for donation and defer those who have who do not qualify. Recommendations for deferral include 1) permanent residents of countries in which malaria is not endemic should be deferred for 1 year after travel to a country in which malaria is endemic, 2) persons with a history of malaria illness should be deferred for 3 years after they are free of symptoms, and 3) persons who lived for $\geq 5$ years in a country with endemic malaria should be deferred for 3 years before becoming eligible for blood donation, and they must have remained free from malaria (and symptoms suggestive of malaria) during that time. Previous residents of countries with endemic malaria who have lived in countries without endemic malaria for at least 3 consecutive years should be deferred for 1 year if they travel to an endemic country, and the other criteria also must be satisfied (68). The 3-year deferral recommendation for residents of countries with endemic malaria is based on data from 1963-1999, which showed that among 4,229 cases of malaria in non-U.S residents, seven patients $(0.2 \%)$ experienced onset of malaria symptoms $>3$ years after they had left the country where malaria is endemic $(68,69)$.

In the 2016 case, the identified donor lived in sub-Saharan Africa for approximately 17 years and reported having malaria regularly during that time. However, the donor had subsequently lived in the United States for approximately 16 years, and despite frequent travel for work and to visit friends and relatives in Africa, most recently in early 2015, her donation was accepted in accordance with the policy because it had been $>1$ year since she had last traveled to an country with endemic malaria, she was asymptomatic, and her last malaria illness was $>15$ years earlier. This donor has characteristics similar to those of other donors who transmitted malaria through transfusion: she was asymptomatic, she was a former resident of a country with endemic malaria, and her responses to the donor questionnaire satisfied the donor eligibility criteria (69-72). Although the risk for contracting malaria from a blood transfusion is very low, a history of blood transfusion should prompt consideration of malaria as a cause of febrile illness.

In cooperation with CDC, state and local health departments investigated three unresolved cryptic cases of $P$. falciparum malaria in 2016. Two of these patients were siblings living in the same household, and all three patients were former residents of a country with endemic malaria. The two siblings were from Honduras, and the third patient was a woman who had been born in Liberia. Rather than relying only on self-report to validate travel history, investigators used a passport review for two of the three cases. In addition, medical records were available that documented the prenatal care of the female sibling in the months before her infection, making it unlikely that she had traveled $<45$ days before illness onset. Except for the siblings, no epidemiological link to another imported 
case was found. The possibility of ongoing local transmission was evaluated for all three cryptic cases, and no additional case of malaria or fever of unknown origin was identified; mosquito surveillance data supported that the risk for ongoing transmission was low. Explanations for the cryptic cases could include nondisclosed travel, a late recurrence from previous infections, or local transmission in the United States from an unidentified infected person.

Both the donor in the transfusion-transmitted malaria case and the female sibling from Honduras were pregnant immediately before illness onset or within 7 months of blood donation. Case studies have shown late recurrences of malaria parasitemia among pregnant women $(73,74)$. In addition, a case-control study showed that late recurrences of $P$. falciparum are more common among pregnant women, HIV-positive patients, and first-arrival immigrants (75) than among others. One hypothesis is that very low parasitemia infections are controlled by acquired immunity, and pregnancy-induced immunosuppression results in the recurrence of the parasites to detectable levels. Another mechanism, which was initially suggested to explain why primigravid women living in Africa are more susceptible to malaria than multigravid women despite acquired immunity, is that parasites can trigger an antigenic shift in the placenta, resulting in the inability of acquired immunity to control the infection, especially among primigravid pregnant women in the second and third trimester (76-78). Although no direct evidence exists, pregnancy might have induced a late recurrence of parasitemia in these two women years after a previous malaria illness. Then each woman became a source of transmission for the transfusiontransmitted malaria and perhaps local transmission of malaria to a sibling living in the same household. Nondisclosed travel, an inaccurate clinical history, or local transmission from an unidentified parasitemic person could also explain these events.

Combining the 2015 and 2016 analysis years, limited by small sample size (52 pregnant $P$. falciparum cases out of 2,045 total $P$. falciparum cases), the frequency of late recurrences among pregnant women compared with nonpregnant women was analyzed. The frequency of late $P$. falciparum recurrences among pregnant women (four of 52 [7.7\%]) compared with nonpregnant women (60 of 2,045 [3.0\%]) was not significantly different. Patients without an onset date (including those who are asymptomatic) or return travel date were excluded from the late recurrence analysis. Because the donor in the transfusion-transmitted malaria case was asymptomatic and was not pregnant at the time of diagnosis, she was not included in the late recurrence analysis.

Health care providers should always consider malaria in the differential diagnosis for a fever of unknown origin, especially among persons who originated from a country with endemic malaria, even if no recent travel is reported. CDC and public health officials have recently investigated several potential cryptic cases among patients who did not disclose their travel history completely or accurately (CDC, unpublished data, 2018). In the vast majority of potential cryptic cases, nondisclosed travel was discovered; sometimes a passport review or an inquiry into official travel records can be helpful to identify nondisclosed travel, especially after the Ebola virus epidemic because some persons might be attempting to avoid the consequences of reporting travel to certain countries.

Chemoprophylaxis is the most effective way for U.S. residents to prevent malaria during travel to a country where malaria is endemic. Lack of chemoprophylaxis use is prevalent among persons who receive a diagnosis of malaria in the United States. Because pretravel health consultations and antimalarial medications for chemoprophylaxis often are not covered by insurance, out-of-pocket costs could prevent U.S. travelers from initiating chemoprophylaxis (79). Barriers to adherence include forgetfulness, lack of awareness about the disease and its potential severity $(80-84)$, and adverse effects (actual or perceived) $(83,84)$. International travelers are heterogeneous, with different motivations, levels of education, and barriers to chemoprophylaxis use. They include both short-stay travelers (e.g., tourists and airline crew members) and long-term travelers (e.g., Peace Corps volunteers, missionaries, disaster and relief workers, and military personnel). Chemoprophylaxis regimens must be tailored according to age, pregnancy status, destination country, patient preferences (e.g., daily or weekly drug regimen), tolerability of potential side effects (e.g., sun sensitivity for doxycycline and neuropsychiatric disorders for mefloquine), and cost. However, persons who are educated about the risk for malaria illness and the safety and effectiveness of malaria chemoprophylaxis can become motivated to take the medication correctly for the duration of their exposure (85-87). Among U.S. residents who received a malaria diagnosis in 2016, traveling to visit friends and relatives was common $(58.9 \%)$, and only $20.0 \%$ of these patients reported any chemoprophylaxis use. Chemoprophylaxis use and adherence among children aged $<18$ years is largely determined by parents or guardians; $50.0 \%$ of U.S. resident children did not take medication to prevent malaria while traveling in countries where malaria is endemic. Health care providers should talk to their patients, especially VFR travelers who might travel to countries where malaria is endemic, about upcoming travel plans and offer education and medication to prevent malaria. Resources for discussing malaria prevention among VFR travelers is available on the CDC website (https:// www.cdc.gov/malaria/travelers/vfr.html).

In 2018, FDA approved tafenoquine (Arakoda) for malaria chemoprophylaxis in nonpregnant persons aged $\geq 18$ years who 
are not G6PD deficient as confirmed by a quantitative G6PD test (88). Arakoda chemoprophylaxis offers protection from acute Plasmodium infections, in addition to protection from relapsing $P$. vivax and $P$. ovale infections. The recommended regimen is a loading dose taken once daily for 3 days before travel (200 mg daily for 3 days), then a weekly dose while traveling in the area where malaria is endemic ( $200 \mathrm{mg} / \mathrm{week})$, followed by a single dose $(200 \mathrm{mg})$ during the week after departing the malaria transmission area. The most common side effects are gastrointestinal disturbances, dizziness, and headache (89). Hemolytic anemia can occur if Arakoda is used by persons with G6PD deficiency, and rare psychiatric adverse reactions were observed in persons with a history of neuropsychiatric illness; hypersensitivity reactions are possible. At higher-than-recommended doses, a vortex keratopathy not affecting vision was observed but resolved within 1 year of stopping the drug (89). Adverse reactions to this new antimalarial should be reported through the FDA MedWatch site (http://www.fda.gov/Safety/MedWatch/default.htm).

UNWTO estimates that during 2010-2030, tourist arrivals will increase at a rate of $4.4 \%$ per year in emerging destinations, including Africa, compared with a 2.2\% increase in traditional destinations like Europe and North America (51). As international travel increases, prevention strategies and health communication messages become even more important for protecting travelers from infectious diseases and reducing risk for importation of acquired illnesses that could result in continual transmission in the United States. Health care providers should discuss malaria prevention strategies with patients who are considering travel to a country with malaria transmission. Although travel can occur at any time of the year, providers should take into consideration that travel most often occurs during the summer and winter holidays; therefore, malaria prevention discussions are most timely in late spring and early summer, accompanied with reminders from late fall through early winter. Travelers should be informed of the risk for malaria and encouraged to use protective measures, especially chemoprophylaxis.

The mainstay of malaria diagnosis is timely microscopy. Thick blood smears are more sensitive than thin smears for detecting malaria parasites because a greater volume of blood is examined in each microscopic field. Thin smears facilitate identification and quantification of the parasite species (90). Blood smears should be read immediately, and the percentage of parasitized red blood cells should be calculated and provided promptly to the treating clinician; qualified personnel who can perform this task should be on call 24 hours per day. Laboratories that can perform a complete blood count with a manual differential have the resources to do a malaria blood smear. CDC provides telediagnosis assistance to laboratories and care providers who need help promptly reading the blood smears (91). In 2017, a follow-up to a 2010 nationwide survey of laboratories was conducted in the United States $(92,93)$. Results showed that although most laboratories offered malaria diagnostic testing services, only $12 \%$ were in complete compliance with all of the Clinical and Laboratory Standards Institute guidelines for analysis and reporting of results in 2017 (92). Limited laboratory experience with malaria specimens can be a barrier to accurate diagnoses, and three fourths of laboratories surveyed reported diagnosing five or fewer cases annually (92). Despite some progress in laboratory performance, compliance with malaria diagnosis recommendations needs improvement. Laboratories unable to provide immediate blood film microscopy should maintain a supply of malaria RDTs to assist with the initial diagnosis of malaria and develop standard procedures to ensure prompt confirmation testing by microscopy or PCR.

PCR testing, which is not as timely, is important for species confirmation and should be used to confirm the results of microscopy and evaluate for mixed infections. In 2014, the Council of State and Territorial Epidemiologists released a revised malaria case definition highlighting the importance of determining the species and the percent parasitemia at the time of diagnosis and encouraging PCR testing for each case (17). If PCR testing is not available locally to confirm the Plasmodium species, then whole blood and blood smears can be sent to CDC for complementary diagnostic confirmation. CDC provides free diagnostic assistance to laboratories and health professionals diagnosing cases of malaria, including microscopy, PCR testing, and drug resistance marker testing (91). Increasing the proportion of cases with species confirmation and drug resistance marker testing will improve understanding of the epidemiology of malaria diagnosed in the United States. Public health laboratories should consider developing standard procedures for sending blood samples from persons with malaria to CDC for molecular surveillance monitoring.

In 2007, FDA approved the BinaxNOW malaria RDT for use in the United States by hospital or commercial laboratories (not for use by clinicians or the public) (94). RDTs allow clinical laboratories that do not have malaria microscopy or PCR capacity to test patients quickly for malaria instead of sending samples off-site for a delayed diagnosis. However, the BinaxNow RDT might not detect malaria in those with very low parasitemia levels, which would have been detected by microscopy, and the test is limited in its ability to identify all Plasmodium species. A microscopy blood smear should be performed for every patient assessed for malaria; good case management requires an assessment of the percent parasitemia to select the appropriate treatment for either uncomplicated or 
severe malaria, which is not possible with RDTs or PCR. For surveillance purposes in the United States, a person who has a positive RDT result is considered to have a suspected malaria case, and microscopy, PCR, or both should be performed to confirm the case (17). In 2016, 10 suspected cases that were only determined by RDT were reported to CDC.

The quality of malaria surveillance data can be limited if the data are incomplete or definitions are incorrectly used. All sections of the malaria case report form should be completed because they provide critical information for examining malaria trends that are used to develop recommendations for malaria treatment and chemoprophylaxis. Local and state health departments, health care providers, and other health personnel should be vigilant in reporting complete information for malaria cases, especially for essential variables including species, residence, and country of acquisition. Incomplete reporting of antimalarial treatments and the infecting species could result in the patient being misclassified for receiving treatment according to CDC guidelines. CDC occasionally receives incomplete records for cases only reported electronically through NNDSS; these records contain basic demographic data but not the additional malaria case information from the NMSS case report form. CDC is collaborating with state and local health departments to update the NNDSS reporting platform through the NNDSS modernization initiative (NMI). Electronic submission of extended malaria data elements through NMI is anticipated to begin in 2019. States that implement malaria reporting via the NMI surveillance system will no longer be required to submit paper case report forms, which should improve the timeliness and accuracy of malaria surveillance in the United States. Additionally, in 2019 CDC is developing an electronic case report form for jurisdictions to use during the transition period to electronic reporting through NMI. State and local health departments are encouraged to report cases using the electronic NMSS case report form until malaria-specific data can be received electronically through NMI. In 2016, despite an increase in the number of confirmed cases compared with 2015, a total of $16(<1.0 \%)$ cases could not be classified, a decrease from $31(2.0 \%)$ unclassified cases in 2015.

In the Caribbean region, transmission of endemic malaria ended in the mid-1960s, except on the island of Hispaniola, which includes the countries of the Dominican Republic and Haiti (95). According to NMSS, an average of 3.1 malaria cases were imported per year from the Dominican Republic during 1995-2014 (36,96). In 2015, nearly half of the 67 cases from Central America and the Caribbean originated from the Dominican Republic but dropped significantly to about 20\% of the 70 imported cases from the region in 2016. According to the Pan American Health Organization, the number of cases detected locally in the Dominican Republic increased from 491 cases in 2014 to 651 cases in 2015 to 722 cases in 2016, and transmission remains in some parts of the country, including the province of La Altagracia, a popular tourist destination $(2,97,98)$. In response to the significant increase in cases imported from the Dominican Republic in 2015, CDC posted a website alert to notify travelers of the numerous malaria cases detected in this area and provided information for prevention, especially chemoprophylaxis (99). None of the 13 patients who traveled to the Dominican Republic in 2016 took chemoprophylaxis for malaria prevention.

Despite the development of chloroquine resistance throughout the world (100-102), chloroquine remains an effective antimalarial for chemoprophylaxis and treatment of P. falciparum malaria acquired from Hispaniola. Monitoring chloroquine resistance markers is recommended (103-107), and health care providers should contact CDC to assist with the evaluation of potential chloroquine failures identified after travel to Hispaniola. Two samples from patients with P. falciparum infections acquired from Hispaniola (one each from the Dominican Republic and Haiti) were tested for molecular resistance markers in Haiti, and none contained a genotype associated with chloroquine resistance.

The number of malaria cases imported from Honduras increased significantly from 2015 to 2016 (four cases in 2015 and 12 cases in 2016), and this trend might reflect an increase in the number of malaria cases detected locally in Honduras $(98,108)$. The number of imported cases from other countries in the Central America and Caribbean regions did not change significantly.

NMSS includes information on military personnel who received a malaria diagnosis in the United States. Before 2010, cases among military patients were only reported to CDC by local and state health departments and private health clinicians. However, starting in 2010, CDC collaborated with the Armed Forces Health Surveillance Branch to facilitate direct reporting, thus improving opportunities to monitor trends or changes in the deployed military population (e.g., changes in geographical transmission and prophylaxis or treatment failures). From 2015 to 2016, no significant change occurred in the proportion of military cases reported among the total cases, although the number increased from 23 cases to 41 cases, respectively. In 2016, an equal number of military cases were acquired in Asia and Africa (17 cases each). Of the military cases acquired in Africa (with known countries of origin), nine were from Central Africa, and five were from West Africa. Of those acquired in Asia, 10 were from South Korea and seven from Afghanistan. Because of the potentially longer interval between date of return to the United States and symptom onset in cases acquired from Asia compared with Africa (mean interval of 
113.4 days versus 11.0 days, respectively), health care providers and military men and women themselves should be aware that symptoms from malaria could occur months or even more than a year after the conclusion of a military deployment in Asia, potentially during a time when the military service member is no longer under the care of the U.S. military medical services.

The U.S. military does not recommend chemoprophylaxis for those deployed to South Korea $(5,48)$. However, chemoprophylaxis is recommended for military members serving elsewhere in countries with malaria transmission. Although rare, persons who adhere to chemoprophylaxis can become infected with malaria. Among the case reports from military patients with information on prophylaxis $(n=14)$, only two reported complete adherence to chemoprophylaxis. This reported nonadherence to chemoprophylaxis is contrary to the successful efforts used to protect military men and women from contracting malaria during the 2015 Ebola virus military response, when approximately 3,000 troops were deployed in 2015, yet only three cases of malaria were diagnosed in the United States among military personnel. This achievement can be attributed to the resources committed to malaria prevention, including chemoprophylaxis with atovaquone-proguanil as the preferential antimalarial, insecticide-treated mosquito nets and tents to protect sleeping spaces, skin repellents, and insecticidetreated uniforms $(87,109)$. In addition, some deployed military personnel were assessed daily for fever and adherence to chemoprophylaxis (110). A survey conducted in Liberia during the Ebola response indicated that $96 \%$ of military personnel reported daily adherence to malaria prophylaxis in addition to other prevention measures; this level of chemoprophylaxis adherence is regarded to be among the highest reported for deployed U.S. military personnel (87). Consequently, when malaria risk awareness is high and chemoprophylaxis and other protection measures are correctly implemented, malaria can be effectively prevented, even among those in high-transmission areas and in stressful deployment situations.

Primaquine use to prevent relapse from $P$. vivax or $P$. ovale in nonpregnant patients remains low (38.7\% among 305 P. vivax or $P$. ovale infections). In 2016, primaquine was the only antimalarial that was active against the dormant liver parasites to prevent relapses. During this time, the treatment recommendation was that all persons who receive a diagnosis of mosquito-acquired P. vivax or P. ovale and who are not G6PD deficient (confirmed by quantitative G6PD testing) should receive a 14-day course of primaquine to prevent relapses $(22,26)$. Adherence to a 14-day regimen can be challenging. In 2018, the FDA approved tafenoquine (Krintafel) for radical cure, also known as antirelapse treatment (111). Tafenoquine is an analog of primaquine, an 8-aminoquinoline, and targets Plasmodium hypnozoites in the liver. Like primaquine, tafenoquine is contraindicated among persons who are G6PD deficient or pregnant. Krintafel is administered as a single 300-mg dose, which might improve adherence to antirelapse malaria treatment. Hemolytic anemia can occur if Krintafel is used by persons with G6PD deficiency, and hypersensitivity reactions are possible (111). At doses higher than the recommended regimen, psychiatric adverse reactions were observed (rarely) in persons with a history of neuropsychiatric illness, and reversible vortex keratopathy not affecting vision was observed (111). Adverse reactions to this new antimalarial should be reported through the FDA MedWatch site (https:// www.fda.gov/safety/medwatch) and through routine malaria case surveillance.

Treatment choices for pregnant women with uncomplicated malaria are limited: mefloquine or quinine plus clindamycin (22). However, in April 2018, CDC released a policy note to recommend artemether-lumefantrine for the treatment of uncomplicated malaria among women in the second and third trimesters of pregnancy. Artemether-lumefantrine should be considered during the first trimester when other treatment options are unavailable (50). This recommendation is consistent with WHO treatment guidelines and reflects current data on treatment efficacy and birth outcomes $(112,113)$.

Since 2000, the case-fatality rate from malaria has been $<1 \%$, with an average of $0.42 \%$. In 2016 , seven deaths from malaria were reported, a case-fatality rate of $0.34 \%$, which was not significantly different from 2015 (11 deaths [0.72\%] casefatality rate). In 2016, four of seven patients who died were infected with $P$. falciparum, two were infected with $P$. vivax, and one had a mixed infection with $P$. falciparum and $P$. vivax. Although severe malaria is best prevented by appropriate administration of chemoprophylaxis, chemoprophylaxis use was not reported by any of the patients who died from malaria.

Once malaria has occurred, prompt initiation of treatment is critical; patients must seek evaluation for fever, health care providers should consider and test for malaria, and appropriate treatment must be provided quickly. Two persons who died sought treatment for their illness 3 days after fever onset, and one patient sought care 5 days after illness onset. Two patients who died sought treatment 1 or 2 days after fever onset; however, their health care providers did not evaluate them for malaria, and the patients were told they had influenza or another respiratory infection. Two patients who died had other symptoms (e.g., diarrhea and lower extremity edema) for weeks before seeking care and did not have a fever, although one patient was febrile when admitted to the hospital. One patient who died from severe $P$. vivax infection returned from a country with endemic malaria 9 months before symptom onset. Because severe malaria can occur quickly, especially in nonimmune persons, when symptoms of malaria occur, 
persons should seek care immediately and notify care providers of their travel history, even if travel was not recent. To facilitate a prompt diagnosis, providers should include malaria in the differential diagnosis of fever in a person who has traveled to a country with malaria transmission or who is originally from an area with endemic malaria. In addition, hospitals should maintain on-site malaria diagnosis capabilities. Signs and symptoms of malaria are often nonspecific but typically include fever. Other symptoms include headache, chills, increased sweating, back pain, myalgia, diarrhea, nausea, vomiting, and cough. Health care providers should ask all febrile patients for a travel history, which is continually important as international travel continues to increase. Patients who have had blood transfusions should be evaluated for malaria if they experience a fever. Any delay in the diagnosis and treatment of malaria can result in complications, regardless of the effectiveness of the treatment regimen. Patients suspected of having a malaria infection should be evaluated with a microscopic examination of thick and thin blood films, as discussed previously (90).

The best approach to prevent severe malaria complications is to provide prompt and appropriate treatment. The choice of an antimalarial treatment regimen should be based on several factors, including the probable geographic origin of the parasite, the Plasmodium species, the percentage parasitemia, and the patient's clinical status $(22,26)$. Oral regimens are less effective than parenteral regimens for treatment of severe malaria, and are not the standard of care. Severely ill patients should be treated aggressively with parenteral antimalarial therapy to ensure rapid adequate drug levels, with the goal to decrease parasitemia to $<1 \%$ as soon as possible to minimize the likelihood of complications or death (22). Quinidine gluconate was the only FDA-approved medication for parenteral malaria therapy in 2016; however, as of April 2019, quinidine gluconate is no longer available in the United States (114). However, intravenous artesunate, the WHO-recommended first-line drug for severe malaria, is available through an investigational new drug protocol from CDC for every patient in the United States with severe malaria (115). Artesunate is stocked in several sites in the United States and can be quickly shipped free of charge to the major airport closest to the requesting hospital. CDC will release artesunate before the required documents have been completed (i.e., the signed consent form and the signed FDA form); however, providers who administer artesunate to patients must comply with the investigational new drug protocol and, after administration of the medication, provide the signed documents, notify CDC of any adverse event, and complete case report forms (115). To obtain artesunate for a patient with severe malaria, health care providers should call the CDC Malaria Hotline at 770-488-7788 or toll-free at 855-856-4713,
Monday-Friday, 9 a.m. -5 p.m., Eastern Time. After hours, callers should call 770-488-7100 and ask to speak with a CDC Malaria Branch clinician. Travelers and health care providers are encouraged to use CDC resources on malaria prevention and treatment (Table 8) and contact the CDC Malaria Branch for assistance with diagnostic or case management needs.

In 2016, patients with severe malaria were significantly more likely to have been inappropriately treated compared with those patients with uncomplicated malaria $(50.3 \%$ versus $9.8 \%$, respectively), and $34.8 \%$ of all pregnant women received treatment inconsistent with the CDC guidelines for treatment. Health care providers should be familiar with prevention, recognition, and treatment of malaria and are encouraged to consult appropriate sources for malaria prevention and treatment recommendations (Table 8). Health care providers should assess the diagnostic and treatment resources present in their facilities, including availability at night or on weekends. A repeated evaluation of malaria diagnosis capabilities among U.S. laboratories demonstrated that although malaria diagnostic testing services were available to the majority of U.S. laboratories surveyed, few were complying with all of the current guidelines (92). To maintain and improve malaria and other parasitic disease diagnosis capabilities in the United States, CDC conducts training courses (https://www. cdc.gov/dpdx/index.html). CDC provides several resources for health care providers; care providers seeking assistance with diagnosis (including telediagnosis) or treatment of patients with suspected or confirmed malaria should call CDC's Malaria Hotline at 770-488-7788 or toll-free 855-856-4713 during regular business hours or CDC's Emergency Operations Center at 770-488-7100 during evenings, weekends, and holidays (ask to speak to the person on call for the Malaria Branch), or go to CDC's website at https://www.cdc.gov/malaria/ diagnosis_treatment/index.html.

Even though malaria is not endemic in the United States, malaria causes illness and deaths in this country, and recent cases are at an all-time high. Imported cases of malaria can reintroduce Plasmodium parasites into receptive areas (12-14) where the disease is not endemic but potential vectors are present and environmental conditions can support the parasite lifecycle (9). Competent Anopheles mosquitoes and conditions that are conducive to malaria transmission exist in the United States (116). The most effective approach for U.S. residents to prevent malaria is to take chemoprophylaxis medication during travel to a country where the disease is endemic. Detailed recommendations for preventing malaria are available to the general public 24 hours per day online at https://wwwnc.cdc.gov/travel/yellowbook/2018/infectiousdiseases-related-to-travel/malaria. In 2019, a new medication for chemoprophylaxis tafenoquine (Arakoda) is available in 
TABLE 8. Sources for malaria prophylaxis, diagnosis, and treatment recommendations

\begin{tabular}{|c|c|c|c|}
\hline $\begin{array}{l}\text { Type of } \\
\text { information }\end{array}$ & Source & Availability & Contact information \\
\hline \multirow[t]{3}{*}{ Prophylaxis } & $\begin{array}{l}\text { CDC Traveler's Health } \\
\text { Internet site (includes } \\
\text { online access to } \\
\text { Health Information for } \\
\text { International Travel) }\end{array}$ & 24 hours/day & https://wwwnc.cdc.gov/travel \\
\hline & $\begin{array}{l}\text { Health Information for } \\
\text { International Travel } \\
\text { (Yellow Book) }\end{array}$ & $\begin{array}{l}\text { The latest edition is available for sale } \\
\text { from Oxford University Press } \\
\text { (https://global.oup.com/ } \\
\text { academic/?lang=en\&cc=us) } \\
\text { and from major online booksellers. }\end{array}$ & $\begin{array}{l}\text { 800-451-7556 } \\
\text { http://global.oup.com/academic } \\
\text { https://wwwnc.cdc.gov/travel/page/yellowbook-home }\end{array}$ \\
\hline & $\begin{array}{l}\text { CDC Malaria Branch } \\
\text { website with malaria } \\
\text { information and } \\
\text { prophylaxis, by } \\
\text { country (Red Pages) }\end{array}$ & 24 hours/day & https://www.cdc.gov/malaria/travelers/country_table/a.html \\
\hline \multirow[t]{2}{*}{ Diagnosis } & $\begin{array}{l}\text { CDC Division of } \\
\text { Parasitic Diseases and } \\
\text { Malaria diagnostic } \\
\text { internet site (DPDx) }\end{array}$ & 24 hours/day & https://www.dpd.cdc.gov/dpdx \\
\hline & $\begin{array}{l}\text { CDC Division of } \\
\text { Parasitic Diseases and } \\
\text { Malaria diagnostic } \\
\text { CD-ROM (DPDx) }\end{array}$ & $\begin{array}{l}\text { Order by e-mail from } \\
\text { CDC Division of Parasitic Diseases and Malaria }\end{array}$ & dpdx@cdc.gov \\
\hline \multirow[t]{2}{*}{ Treatment } & CDC Malaria Branch & 9:00 a.m.-5:00 p.m. Eastern, Monday-Friday & 770-488-7788 or toll-free $855-856-4713^{*}$ \\
\hline & CDC Malaria Branch & $\begin{array}{l}\text { 5:00 p.m.- } 9: 00 \text { a.m. Eastern on weekdays } \\
\text { and all day weekends and holidays }\end{array}$ & $\begin{array}{l}\text { 770-488-7100* } \\
\text { (This number is for the CDC Emergency Operations Center. } \\
\text { Ask staff member to page the person on call for the Malaria Branch.) } \\
\text { https://www.cdc.gov/malaria/diagnosis_treatment/treatment.html }\end{array}$ \\
\hline
\end{tabular}

* These numbers are intended for health care professionals only.

the United States to prevent malaria, and it offers a weekly dosing schedule to adults who are not pregnant or G6PD deficient. Prevention recommendations tailored for each country are available online at https://www.cdc.gov/malaria/ travelers/country_table/a.html. CDC biannually publishes recommendations in Health Information for International Travel (commonly referred to as the Yellow Book), which is available and updated on the CDC Travelers' Health website at https:// www.cdc.gov/Features/TravelersHealth.html (Table 8).

\section{Acknowledgments}

The authors acknowledge the state, territorial, and local health departments; health care providers; laboratories; and the Armed Forces Health Surveillance Branch for providing malaria information to CDC. Laboratory support was provided by the malaria diagnostic reference laboratory and by Dragan Ljolje and Venkatachalam Udhayakumar (molecular surveillance work).

\section{Conflicts of Interest}

No conflicts of interest were reported.

\section{References}

1. Mayxay M, Pukrittayakamee S, Newton PN, White NJ. Mixed-species malaria infections in humans. Trends Parasitol 2004;20:233-40. https://doi.org/10.1016/j.pt.2004.03.006

2. World Health Organization. World malaria report 2017. Geneva, Switzerland: World Health Organization; 2018. https://www.who.int/ malaria/publications/world-malaria-report-2017/en

3. Alonso P, Noor AM. The global fight against malaria is at crossroads. Lancet 2017;390:2532-4. https://doi.org/10.1016/ S0140-6736(17)33080-5

4. Oh MD, Shin H, Shin D, et al. Clinical features of vivax malaria. Am J Trop Med Hyg 2001;65:143-6. https://doi.org/10.4269/ ajtmh.2001.65.143

5. Klein TA, Pacha LA, Lee HC, et al. Plasmodium vivax malaria among U.S. forces Korea in the Republic of Korea, 1993-2007. Mil Med 2009;174:412-8. https://doi.org/10.7205/MILMED-D-01-4608

6. Battle KE, Karhunen MS, Bhatt S, et al. Geographical variation in Plasmodium vivax relapse. Malar J 2014;13:144. https://doi. org/10.1186/1475-2875-13-144

7. Warrell DA, Gilles HM, eds. Essential malariology. 4th ed. London, United Kingdom: Hodder Arnold; 2002. 
8. Sutherland CJ. Persistent parasitism: the adaptive biology of malariae and ovale malaria. Trends Parasitol 2016;32:808-19. https://doi. org/10.1016/j.pt.2016.07.001

9. Kiszewski A, Mellinger A, Spielman A, Malaney P, Sachs SE, Sachs J. A global index representing the stability of malaria transmission. Am J Trop Med Hyg 2004;70:486-98. https://doi.org/10.4269/ ajtmh.2004.70.486

10. Andrews JM, Quinby GE, Langmuir AD. Malaria eradication in the United States. Am J Public Health Nations Health 1950;40:1405-11. https://doi.org/10.2105/AJPH.40.11.1405

11. Cullen KA, Arguin PM. Malaria surveillance-United States, 2011. MMWR Surveill Summ 2013;62(No. SS-5).

12. Filler SJ, MacArthur JR, Parise M, et al. Locally acquired mosquitotransmitted malaria: a guide for investigations in the United States. MMWR Recomm Rep 2006;55(No. RR-13).

13. CDC. Local transmission of Plasmodium vivax malaria-Palm Beach County, Florida, 2003. MMWR Morb Mortal Wkly Rep 2003;52:908-11.

14. CDC. Multifocal autochthonous transmission of malaria-Florida, 2003. MMWR Morb Mortal Wkly Rep 2004;53:412-3.

15. Leder K, Black J, O'Brien D, et al. Malaria in travelers: a review of the GeoSentinel surveillance network. Clin Infect Dis 2004;39:1104-12. https://doi.org/10.1086/424510

16. CDC. National notifiable disease surveillance system. Atlanta, GA: US Department of Health and Human Services, CDC; 2015. https:// wwwn.cdc.gov/nndss/default.aspx

17. Council of State and Territorial Epidemiologists. Public health reporting and national notification for malaria. Atlanta, GA: Council of State and Territorial Epidemiologists; 2014. https://c.ymcdn.com/sites/www. cste.org/resource/resmgr/PS/13-ID-08.pdf

18. BinaxNOW Malaria [package insert]. Scarborough, ME: Inverness Medical Professional Diagnostics; 2007.

19. CDC. Instructions for using the malaria case surveillance report form. Atlanta, GA: US Department of Health and Human Services, CDC. https://www.cdc.gov/malaria/resources/pdf/report/ survellianceformcoverletter2.pdf

20. CDC. Malaria case surveillance report. Atlanta, GA: US Department of Health and Human Services, CDC; 2014. https://www.cdc.gov/ malaria/resources/pdf/report/malaria_form.pdf

21. Arguin PM. A definition that includes first and second generation immigrants returning to their countries of origin to visit friends and relatives still makes sense to me. J Travel Med 2010;17:147-9. https:// doi.org/10.1111/j.1708-8305.2010.00412.x

22. CDC. Guidelines for treatment of malaria in the United States. Atlanta, GA: US Department of Health and Human Services, CDC; 2013. https://www.cdc.gov/malaria/diagnosis_treatment/treatment.html

23. World Health Organization. WHO malaria terminology. Geneva, Switzerland: Global Malaria Programme; 2016. https://www.who.int/ malaria/publications/atoz/malaria-terminology/en

24. CDC. CDC health information for international travel. Atlanta, GA: US Department of Health and Human Services, CDC; 2018. https://wwwnc. cdc.gov/travel/yellowbook/2018/infectious-diseases-related-to-travel/malaria

25. Imwong M, Snounou G, Pukrittayakamee S, et al. Relapses of Plasmodium vivax infection usually result from activation of heterologous hypnozoites. J Infect Dis 2007;195:927-33. https://doi. org/10.1086/512241

26. Griffith KS, Lewis LS, Mali S, Parise ME. Treatment of malaria in the United States: a systematic review. JAMA 2007;297:2264-77. https:// doi.org/10.1001/jama.297.20.2264
27. World Health Organization. Management of severe malaria: a practical handbook. Geneva, Switzerland: WHO Press; 2012.

28. CDC. Preparation of blood smears. Atlanta, GA: US Department of Health and Human Services, CDC; 2013.

29. CDC. Staining for malaria parasites. Atlanta, GA: US Department of Health and Human Services, CDC; 2013. https:/www.cdc.gov/dpdx/ resources/pdf/benchAids/malaria/malaria_staining_benchaid.pdf

30. CDC. Notice to readers: malaria rapid diagnostic test. MMWR Morb Mortal Wkly Rep 2007;56:686.

31. Bacon DJ, McCollum AM, Griffing SM, et al. Dynamics of malaria drug resistance patterns in the Amazon basin region following changes in Peruvian national treatment policy for uncomplicated malaria. Antimicrob Agents Chemother 2009;53:2042-51. https://doi. org/10.1128/AAC.01677-08

32. Korsinczky M, Chen N, Kotecka B, Saul A, Rieckmann K, Cheng Q. Mutations in Plasmodium falciparum cytochrome $\mathrm{b}$ that are associated with atovaquone resistance are located at a putative drug-binding site. Antimicrob Agents Chemother 2000;44:2100-8. https://doi. org/10.1128/AAC.44.8.2100-2108.2000

33. Price RN, Uhlemann AC, Brockman A, et al. Mefloquine resistance in Plasmodium falciparum and increased pfmdr1 gene copy number. Lancet 2004;364:438-47. https://doi.org/10.1016/S0140-6736(04)16767-6

34. Ariey F, Witkowski B, Amaratunga C, et al. A molecular marker of artemisinin-resistant Plasmodium falciparum malaria. Nature 2014;505:50-5. https://doi.org/10.1038/nature12876

35. Talundzic E, Okoth SA, Congpuong K, et al. Selection and spread of artemisinin-resistant alleles in Thailand prior to the global artemisinin resistance containment campaign. PLoS Pathog 2015;11:e1004789. https://doi.org/10.1371/journal.ppat.1004789

36. Mace KE, Arguin PM, Tan KR. Malaria surveillance-United States, 2015. MMWR Surveill Summ 2018;67(No. SS-13). https://doi. org/10.15585/mmwr.ss6707a1

37. National Travel and Tourism Office. International air travel statistics (I-92 data), 1996-2017. Washington, DC: National Travel and Tourism Office. https://travel.trade.gov/research/monthly/departures/index.asp

38. CDC. Overseas refugee health guidelines: malaria. Atlanta, GA: US Department of Health and Human Services, CDC; 2012. https://www. cdc.gov/immigrantrefugeehealth/pdf/malaria-overseas.pdf

39. Mali S, Steele S, Slutsker L, Arguin PM. Malaria surveillanceUnited States, 2008. MMWR Surveill Summ 2010;59(No. SS-7).

40. Mali S, Tan KR, Arguin PM. Malaria surveillance-United States, 2009. MMWR Surveill Summ 2011;60(No. SS-3).

41. Mali S, Kachur SP, Arguin PM. Malaria surveillance-United States, 2010. MMWR Surveill Summ 2012;61(No. SS-2).

42. Cullen KA, Arguin PM. Malaria surveillance-United States, 2012. MMWR Surveill Summ 2014;63(No. SS-12).

43. Cullen KA, Mace KE, Arguin PM. Malaria surveillance-United States, 2013. MMWR Surveill Summ 2016;65(No. SS-2). https://doi. org/10.15585/mmwr.ss6502a 1

44. Mace KE, Arguin PM. Malaria surveillance- United States, 2014. MMWR Surveill Summ 2017;66(No. SS-12). https://doi. org/10.15585/mmwr.ss6612a1

45. CDC. As West Africa Ebola outbreak worsens, CDC issues level 3 travel warning. CDC surge scaling up response in Guinea, Sierra Leone, and Liberia. Atlanta, GA: US Department of Health and Human Services, CDC; 2014.

46. Cohen NJ, Brown CM, Alvarado-Ramy F, et al. Travel and border health measures to prevent the international spread of Ebola. MMWR Suppl 2016;65(No. Suppl 3). https://doi.org/10.15585/mmwr.su6503a9 
47. CDC. Notes on the interim U.S. guidance for monitoring and movement of persons with potential Ebola virus exposure. Atlanta, GA: US Department of Health and Human Services, CDC; 2016. https://www.cdc.gov/vhf/ebola/exposure/monitoring-and-movementof-persons-with-exposure.html

48. Kim HC, Pacha LA, Lee WJ, et al. Malaria in the Republic of Korea, 1993-2007. Variables related to re-emergence and persistence of Plasmodium vivax among Korean populations and U.S. forces in Korea. Mil Med 2009;174:762-9. https://doi.org/10.7205/ MILMED-D-01-6208

49. CDC. New medication for severe malaria available under an investigational new drug protocol. MMWR Morb Mortal Wkly Rep 2007;56:769-73.

50. Ballard SB, Salinger A, Arguin PM, Desai M, Tan KR. Updated CDC recommendations for using artemether-lumefantrine for the treatment of uncomplicated malaria in pregnant women in the United States. MMWR Morb Mortal Wkly Rep 2018;67:424-31. https://doi. org/10.15585/mmwr.mm6714a4

51. World Tourism Organization. UNWTO tourism highlights, 2017 edition. Madrid, Spain: World Tourism Organization; 2017.

52. World Tourism Organization. UNWTO tourism highlights, 2018 edition. Madrid, Spain: World Tourism Organization; 2018.

53. Angelo KM, Libman M, Caumes E, et al; GeoSentinel Network. Malaria after international travel: a GeoSentinel analysis, 2003-2016. Malar J 2017;16:293. https://doi.org/10.1186/s12936-017-1936-3

54. Tatem AJ, Jia P, Ordanovich D, et al. The geography of imported malaria to non-endemic countries: a meta-analysis of nationally reported statistics. Lancet Infect Dis 2016;17:98-107. PubMed

55. Pan American Health Organization. Timeline of emergence of Zika virus in the Americas. Geneva, Switzerland: World Health Organization; 2016. https://www.paho.org/hq/index.php?option=com_content \& view=article\&id=11959:timeline-of-emergence-of-zika-virus-in-theamericas\&Itemid $=41711$ \&lang=en

56. Zhang Q, Sun K, Chinazzi M, et al. Spread of Zika virus in the Americas. Proc Natl Acad Sci U S A 2017;114:E4334-43. https://doi. org/10.1073/pnas.1620161114

57. World Health Organization WHO Director-General summarizes the outcome of the emergency committee regarding clusters of microcephaly and Guillain-Barré syndrome. Geneva, Switzerland: World Health Organization; 2016. https://www.who.int/news-room/ detail/01-02-2016-who-director-general-summarizes-the-outcome-ofthe-emergency-committee-regarding-clusters-of-microcephaly-andguillain-barr\%C3\%A9-syndrome

58. CDC. CDC concludes Zika causes microcephaly and other birth defects. Atlanta, GA: US Department of Health and Human Services, CDC; 2016. https://www.cdc.gov/media/releases/2016/s0413-zikamicrocephaly.html

59. CDC. Zika travel information. Atlanta, GA: US Department of Health and Human Services, CDC; 2018. https://wwwnc.cdc.gov/travel/page/ zika-travel-information

60. CDC. Zika virus prevention and transmission. Atlanta, GA: US Department of Health and Human Services, CDC; 2018. https:// www.cdc.gov/zika/prevention/index.html

61. Maroushek SR, Aguilar EF, Stauffer W, Abd-Alla MD. Malaria among refugee children at arrival in the United States. Pediatr Infect Dis J 2005;24:450-2. https://doi.org/10.1097/01. inf.0000160948.22407.0d
62. Ndao M, Bandyayera E, Kokoskin E, Gyorkos TW, MacLean JD, Ward BJ. Comparison of blood smear, antigen detection, and nestedPCR methods for screening refugees from regions where malaria is endemic after a malaria outbreak in Quebec, Canada. J Clin Microbiol 2004;42:2694-700. https://doi.org/10.1128/ JCM.42.6.2694-2700.2004

63. Lindblade KA, Steinhardt L, Samuels A, Kachur SP, Slutsker L. The silent threat: asymptomatic parasitemia and malaria transmission. Expert Rev Anti Infect Ther 2013;11:623-39. https://doi.org/10.1586/ eri. 13.45

64. Bousema T, Okell L, Felger I, Drakeley C. Asymptomatic malaria infections: detectability, transmissibility and public health relevance. Nat Rev Microbiol 2014;12:833-40. https:/doi.org/10.1038/ nrmicro3364

65. Groger M, Veletzky L, Lalremruata A, et al. Prospective clinical trial assessing species-specific efficacy of artemether-lumefantrine for the treatment of Plasmodium malariae, Plasmodium ovale, and mixed Plasmodium malaria in Gabon. Antimicrob Agents Chemother 2018;62:e01758-17. https://doi.org/10.1128/AAC.01758-17

66. Collins WE, Jeffery GM. Plasmodium malariae: parasite and disease. Clin Microbiol Rev 2007;20:579-92. https://doi.org/10.1128/ CMR.00027-07

67. Franken G, Müller-Stöver I, Holtfreter MC, et al. Why do Plasmodium malariae infections sometimes occur in spite of previous antimalarial medication? Parasitol Res 2012;111:943-6. https://doi.org/10.1007/ s00436-012-2851-8

68. Food and Drug Administration. Guidance for industry, recommendations for donor questioning, deferral, reentry and product management to reduce the risk of transfusion-transmitted malaria. Washington, DC: US Department of Health and Human Services, Food and Drug Administration; 2014. https:/www.fda.gov/downloads/ BiologicsBloodVaccines/GuidanceComplianceRegulatoryInformation/ Guidances/Blood/ucm080784.pdf

69. Mungai M, Tegtmeier G, Chamberland M, Parise M. Transfusiontransmitted malaria in the United States from 1963 through 1999. N Engl J Med 2001;344:1973-8. https://doi.org/10.1056/ NEJM200106283442603

70. Holtzclaw A, Mrsic Z, Managbanag J, Calvano T, Colombo C. Transfusion-transmitted malaria not preventable by current blood donor screening guidelines: a case report. Transfusion 2016;56:2221-4. https://doi.org/10.1111/trf.13680

71. O’Brien SF, Delage G, Seed CR, et al. The epidemiology of imported malaria and transfusion policy in 5 nonendemic countries. Transfus Med Rev 2015;29:162-71. https://doi.org/10.1016/j.tmrv.2015.03.004

72. Anand A, Mace KE, Townsend RL, et al. Investigation of a case of suspected transfusion-transmitted malaria. Transfusion 2018;58:2115-21. https://doi.org/10.1111/trf.14778

73. Giobbia M, Tonon E, Zanatta A, Cesaris L, Vaglia A, Bisoffi Z. Late recrudescence of Plasmodium falciparum malaria in a pregnant woman: a case report. Int J Infect Dis 2005;9:234-5. https://doi.org/10.1016/j. ijid.2004.08.002

74. Odolini S, Apostoli A, Casari S, Matteelli A, Castelli F. Recrudescence of Plasmodium falciparum malaria in a primigravid woman with anaemia as the only sign of disease. J Obstet Gynaecol 2014;34:356. https:// doi.org/10.3109/01443615.2013.866079

75. D’Ortenzio E, Godineau N, Fontanet A, et al. Prolonged Plasmodium falciparum infection in immigrants, Paris. Emerg Infect Dis 2008;14:323-6. https://doi.org/10.3201/eid1402.061475 
76. Hviid L, Staalsoe T. Late recrudescence of Plasmodium falciparum malaria in pregnancy. Int J Infect Dis 2006;10:412. https://doi. org/10.1016/j.ijid.2005.10.013

77. Ricke CH, Staalsoe T, Koram K, et al. Plasma antibodies from malaria-exposed pregnant women recognize variant surface antigens on Plasmodium falciparum-infected erythrocytes in a parity-dependent manner and block parasite adhesion to chondroitin sulfate A.J Immunol 2000;165:3309-16. https://doi.org/10.4049/jimmunol.165.6.3309

78. Staalsoe T, Shulman CE, Bulmer JN, Kawuondo K, Marsh K, Hviid L. Variant surface antigen-specific IgG and protection against clinical consequences of pregnancy-associated Plasmodium falciparum malaria. Lancet 2004;363:283-9. https://doi.org/10.1016/ S0140-6736(03)15386-X

79. Schultz JS, Atherly AJ, Henao-Martínez AF. A deadly wait for U.S. health insurance coverage-sitting on the couch with malaria. Am J Trop Med Hyg 2018;99:24-6. https://doi.org/10.4269/ajtmh.18-0010

80. Baggett HC, Graham S, Kozarsky PE, et al. Pretravel health preparation among U.S. residents traveling to India to VFRs: importance of ethnicity in defining VFRs. J Travel Med 2009;16:112-8. https://doi. org/10.1111/j.1708-8305.2008.00284.x

81. Balaban V, Warnock E, Ramana Dhara V, Jean-Louis LA, Sotir MJ, Kozarsky P. Health risks, travel preparation, and illness among public health professionals during international travel. Travel Med Infect Dis 2014;12:349-54. https://doi.org/10.1016/j.tmaid.2014.01.007

82. Landman KZ, Tan KR, Arguin PM. Adherence to malaria prophylaxis among Peace Corps volunteers in the Africa region, 2013. Travel Med Infect Dis 2015;13:61-8. https://doi.org/10.1016/j.tmaid.2014.12.001

83. Selent M, de Rochars VM, Stanek D, et al. Malaria prevention knowledge, attitudes, and practices (KAP) among international flying pilots and flight attendants of a US commercial airline. J Travel Med 2012;19:366-72. https://doi.org/10.1111/j.1708-8305.2012.00655.x

84. Brisson M, Brisson P. Compliance with antimalaria chemoprophylaxis in a combat zone. Am J Trop Med Hyg 2012;86:587-90. https://doi. org/10.4269/ajtmh.2012.11-0511

85. Itoh M, Arguin PM. A conversation about chemoprophylaxis. Travel Med Infect Dis 2016;14:434-5. https://doi.org/10.1016/j. tmaid.2016.07.007

86. Tuck J, Williams J. Malaria protection in Sierra Leone during the Ebola outbreak 2014/15; The UK military experience with malaria chemoprophylaxis Sep 14-Feb 15. Travel Med Infect Dis 2016;14:471-4. https://doi.org/10.1016/j.tmaid.2016.07.005

87. Vento TJ, Cardile AP, Littell CT, et al. Compliance with malaria preventive measures by U.S. military personnel deployed in support of Ebola control efforts in Liberia. San Diego, CA: Open Forum Infectious Diseases, IDWeek; 2015. https://academic.oup.com/ofid/article/2/ suppl_1/1609/2634209

88. US Food and Drug Administration approves Arakoda (tafenoquine) tablets for oral use; first preventative antimalarial approved in almost two decades; 2018. https://www.accessdata.fda.gov/drugsatfda_docs/ label/2018/210607lbl.pdf

89. Sixty Degrees Pharmaceuticals. Arakoda (tafenoquine) tablets, for oral use. Washington, DC; Sixty Degrees Pharmaceuticals; 2018. https:// www.accessdata.fda.gov/drugsatfda_docs/label/2018/210607lbl.pdf

90. CDC. Malaria diagnosis (United States). Atlanta, GA: US Department of Health and Human Services, CDC; 2015. https://www.cdc.gov/ malaria/diagnosis_treatment/diagnosis.html
91. CDC. DPDx - Laboratory identification of parasitic diseases of public health concern. Diagnostic assistance. Atlanta, GA: US Department of Health and Human Services, CDC; 2018. https:/www.cdc.gov/dpdx/ dxassistance.html

92. Prestel C, Tan KR, Abanyie F, Jerris R, Gutman JR. Malaria diagnostic practices in U.S. laboratories in 2017. J Clin Microbiol 2018;56:e00461-18. https://doi.org/10.1128/JCM.00461-18

93. Abanyie FA, Arguin PM, Gutman J. State of malaria diagnostic testing at clinical laboratories in the United States, 2010: a nationwide survey. Malar J 2011;10:340. https://doi.org/10.1186/1475-2875-10-340

94. CDC. Malaria diagnosis (U.S.) — rapid diagnostic test. Atlanta, GA: US Department of Health and Human Services, CDC; 2014. https:// www.cdc.gov/malaria/diagnosis_treatment/rdt.html

95. Pan American Health Organization. Status of malaria eradication in the Americas, 18th report. PAHO CSP 18/7. Geneva, Switzerland: World Health Organization; 1970.

96. CDC. National malaria surveillance system. Atlanta, GA: US Department of Health and Human Services, CDC; 2018.

97. Pan American Health Organization. World malaria report 2016. Geneva, Switzerland: World Health Organization; 2017.

98. Pan American Health Organization. Interactive malaria statistics. Geneva, Switzerland: World Health Organization; 2017. http:// www.paho.org/hq/index.php?option=com_content $\&$ view=article \&id=2632:2010-interactive-malaria-statistics\&Itemid=2130\&lang=en

99. CDC. Malaria prevention for travelers to the Dominican Republic. Atlanta, GA: US Department of Health and Human Services, CDC; 2015. https://wwwdev.cdc.gov/malaria/new_info/2015/malariadom_ rep_july2015.htm

100. Blasco B, Leroy D, Fidock DA. Antimalarial drug resistance: linking Plasmodium falciparum parasite biology to the clinic. Nat Med 2017;23:917-28. https://doi.org/10.1038/nm.4381

101. Bloland P. Drug Resistance in Malaria. Geneva, Switzerland: World Health Organization2001.

102. Wongsrichanalai C, Pickard AL, Wernsdorfer WH, Meshnick SR. Epidemiology of drug-resistant malaria. Lancet Infect Dis 2002;2:209-18. https://doi.org/10.1016/S1473-3099(02)00239-6

103. Londono BL, Eisele TP, Keating J, et al. Chloroquine-resistant haplotype Plasmodium falciparum parasites, Haiti. Emerg Infect Dis 2009;15:735-40. https://doi.org/10.3201/eid1505.081063

104. Gharbi M, Pillai DR, Lau R, et al; French National Reference Center for Imported Malaria Study. Chloroquine-resistant malaria in travelers returning from Haiti after 2010 earthquake. Emerg Infect Dis 2012;18:1346-9. https://doi.org/10.3201/eid1808.111779

105. Neuberger A, Zhong K, Kain KC, Schwartz E. Lack of evidence for chloroquine-resistant Plasmodium falciparum malaria, Leogane, Haiti. Emerg Infect Dis 2012;18:1487-9. https://doi.org/10.3201/ eid1809.120605

106. Elbadry MA, Existe A, Victor YS, et al. Survey of Plasmodium falciparum multidrug resistance- 1 and chloroquine resistance transporter alleles in Haiti. Malar J 2013;12:426. https://doi. org/10.1186/1475-2875-12-426

107. Morton LC, Huber C, Okoth SA, et al. Plasmodium falciparum drugresistant haplotypes and population structure in postearthquake Haiti, 2010. Am J Trop Med Hyg 2016;95:811-6. https://doi.org/10.4269/ ajtmh.16-0214

108. Pan American Health Organization. Epidemiological update, increase of malaria in the Americas. Geneva, Switzerland: World Health Organization; 2018. 
109. Woods VM. Biggest threat to U.S. troops in Liberia is malaria, not Ebola. Washington, DC: US Department of Defense, US Army; 2014. https://www.army.mil/article/139340/ biggest_threat_to_us_troops_in_liberia_is_malaria_not_ebola

110. Cardile AP, Murray CK, Littell CT, et al. Monitoring exposure to Ebola and health of U.S. military personnel deployed in support of Ebola control efforts-Liberia, October 25, 2014-February 27, 2015. MMWR Morb Mortal Wkly Rep 2015;64:690-4.

111. GlaxoSmithKline. Krintafel (tafenoquine) tablets, for oral use. Research Triangle Park, NC: GlaxoSmithKline; 2018. https://www.accessdata. fda.gov/drugsatfda_docs/label/2018/210795s000lbl.pdf

112. World Health Organization GMP. Intermittent screening and treatment in pregnancy and the safety of ACTs in the first trimester. 2015 [updated November 2015]; https://www.who.int/malaria/publications/atoz/ istp-and-act-in-pregnancy.pdf?ua $=1$
113. World Health Organization. Guidelines for the treatment of malaria. 3rd ed. Geneva, Switzerland: World Health Organization; 2015. https:// www.who.int/malaria/publications/atoz/9789241549127/en

114. CDC. Quinidine availability in the United States. US Department of Health and Human Services, CDC; 2017. https://www.cdc.gov/ malaria/new_info/2017/Quinidine_2017.html

115. CDC. Notice to readers: new medication for severe malaria available under an investigational new drug protocol. MMWR Morb Mortal Wkly Rep 2007;56:769-70.

116. Levine RS, Peterson AT, Benedict MQ. Distribution of members of Anopheles quadrimaculatus say s.l. (Diptera: Culicidae) and implications for their roles in malaria transmission in the United States. J Med Entomol 2004;41:607-13. https://doi.org/10.1603/0022-2585-41.4.607 


The Morbidity and Mortality Weekly Report (MMWR) Series is prepared by the Centers for Disease Control and Prevention (CDC) and is available free of charge in electronic format. To receive an electronic copy each week, visit MMWR at https://www.cdc.gov/mmwr/index.html.

Readers who have difficulty accessing this PDF file may access the HTML file at https://www.cdc.gov/mmwr/volumes/68/ss/ss6805a1.htm?s_ cid=ss6805a1_w. Address all inquiries about the $M M W R$ Series, including material to be considered for publication, to Executive Editor, $M M W R$ Series, Mailstop E-90, CDC, 1600 Clifton Rd., N.E., Atlanta, GA 30329-4027 or to mmwrq@cdc.gov.

All material in the MMWR Series is in the public domain and may be used and reprinted without permission; citation as to source, however, is appreciated. MMWR and Morbidity and Mortality Weekly Report are service marks of the U.S. Department of Health and Human Services.

Use of trade names and commercial sources is for identification only and does not imply endorsement by the U.S. Department of Health and Human Services.

References to non-CDC sites on the Internet are provided as a service to $M M W R$ readers and do not constitute or imply endorsement of these organizations or their programs by CDC or the U.S. Department of Health and Human Services. CDC is not responsible for the content of these sites. URL addresses listed in $M M W R$ were current as of the date of publication.

ISSN: 0149-2195 (Print) 